\title{
Dark Matter Cosmology and Astrophysics
}

\author{
Vladimir S. Netchitailo \\ Biolase Inc., Irvine, CA, USA \\ Email: v.netchitailo@sbcglobal.net
}

How to cite this paper: Netchitailo, V.S. (2019) Dark Matter Cosmology and Astrophysics. Journal of High Energy Physics, Gravitation and Cosmology, 5, 999-1050. https://doi.org/10.4236/jhepgc.2019.54056

Received: June 27, 2019

Accepted: September 9, 2019

Published: September 12, 2019

Copyright $\odot 2019$ by author(s) and Scientific Research Publishing Inc. This work is licensed under the Creative Commons Attribution International License (CC BY 4.0).

http://creativecommons.org/licenses/by/4.0/

\begin{abstract}
Hypersphere World-Universe Model (WUM) envisions Matter carried from Universe into World from fourth spatial dimension by Dark Matter Particles (DMPs). Luminous Matter is byproduct of Dark Matter (DM) annihilation. WUM introduces Dark Epoch (spanning from Beginning of World for 0.4 billion years) when only DMPs existed, and Luminous Epoch (ever since for 13.8 billion years). Big Bang discussed in standard cosmological model is, in our view, transition from Dark Epoch to Luminous Epoch due to Rotational Fission of Overspinning DM Supercluster's Cores and annihilation of DMPs. WUM solves a number of physical problems in contemporary Cosmology and Astrophysics through DMPs and their interactions: Angular Momentum problem in birth and subsequent evolution of Galaxies and Extrasolar systems-how do they obtain it; Fermi Bubbles-two large structures in gamma-rays and X-rays above and below Galactic center; Mysterious Star KIC $\mathbf{8 4 6 2 8 5 2}$ with irregular dimmings; Coronal Heating problem in solar physics-temperature of Sun's corona exceeding that of photosphere by millions of degrees; Cores of Sun and Earth rotating faster than their surfaces; Diversity of Gravitationally-Rounded Objects in Solar system and their Internal Heat; Lightning Initiation problem-electric fields observed inside thunderstorms are not sufficient to initiate sparks; Terrestrial Gamma-Ray Flashes-bursts of high energy X-rays and gamma rays emanating from Earth. Model makes predictions pertaining to Masses of DMPs, proposes New Types of their Interactions. WUM reveals Inter-Connectivity of Primary Cosmological Parameters and calculates their values, which are in good agreement with the latest results of their measurements.
\end{abstract}

\section{Keywords}

Hypersphere World-Universe Model, Law of Conservation of Angular Momentum, Dark Epoch, Rotational Fission, Luminous Epoch, Multiworld, Dark Matter Particles, Macroobject Shell Model, Dark Matter Core, Medium of the World, Mysterious Star KIC 8462852, Dark Matter Fermi Bubbles, Solar Corona, Geocorona, Planetary Corona, Galactic Wind, Solar Wind, 
High-Energy Atmospheric Physics, Lightning Initiation Problem, Terrestrial Gamma-Ray Flashes, Gamma-Ray Bursts, Gravitational Bursts, Ball Lightning

\section{Introduction}

Hypersphere World-Universe Model (WUM) is proposed as an alternative to the prevailing Big Bang Model of standard physical cosmology. WUM is a classical model, and is described by classical notions, which define emergent phenomena. By definition, an emergent phenomenon is a property that is a result of simple interactions that work cooperatively to create a more complex interaction. Physically, simple interactions occur at a microscopic level, and the collective result can be observed at a macroscopic level. WUM introduces classical notions once the very first ensemble of particles has been created at the cosmological time $\cong 10^{-18} \mathrm{~s}$ (state of the World at cosmological times $<10^{-18} \mathrm{~s}$ is best described by Quantum Mechanics). WUM is a natural continuation of Classical Physics.

The Hypersphere World-Universe model is the only cosmological model in existence that:

- Is consistent with the Law of conservation of angular momentum, and answers the following questions: why is the orbital momentum of Jupiter larger than rotational momentum of Sun, and how did Milky Way galaxy and Solar system obtain their substantial orbital angular momentum?

- Reveals the Inter-connectivity of primary cosmological parameters of the World (Age, Size, Hubble's parameter, Newtonian parameter of gravitation, Critical energy density, Concentration of Intergalactic Plasma, Temperature of the Microwave Background Radiation, Temperature of the Far-Infrared Background Radiation peak) and calculates their values, which are in good agreement with experimental results;

- Considers Fermi Bubbles (FBs) built up from Dark Matter Particles (DMPs), and explains X-rays and gamma-rays radiated by FBs as a result of DMPs annihilation;

- Solves Coronal heating problem that relates to the question of why the temperature of the Solar corona is millions of degrees higher than that of the photosphere. In WUM, the Solar corona is made up of DMPs, and the plas$\mathrm{ma}$ is the result of their annihilation. The Solar corona resembles a honeycomb filled with plasma. The Geocorona and Planetary Coronas possess features similar to these of the Solar Corona;

- Explains the diversity of Very High Energy gamma-ray sources in the World in frames of the proposed Macroobject (MO) Shell Model, which describes Cores of MOs as Nuclei made up of annihilating Dark Matter Fermions (DMFs) surrounded by shells containing other DMPs;

- Explains the diversity of gravitationally-rounded objects (planets and moons 
in Solar system) and their internal heat through annihilation of DMFs in their Cores.

WUM paints the following picture of creation and evolution of the World:

- Overspinning (surface speed at equator exceeding escape velocity) DM Cores of Superclusters initiate creation of all World's Macrostructures;

- The outer shells of Supercluster's Cores are composed of DMFs named Dions, with mass of $0.2 \mathrm{eV}$ and total energy density of $68.8 \%$ of the overall energy density of the World;

- Proposed Weak Interaction between DMPs provides the integrity of Dark Matter (DM) Cores of all MOs;

- Dions' outer shells of Supercluster's Cores are growing to the critical mass during Dark Epoch lasting from the Beginning of the World (14.2 billion years ago) for 0.4 billion years;

- Luminous Galaxies and Extrasolar Systems arise due to Rotational Fission of Overspinning Supercluster's Cores and annihilation of DMPs;

- Macrostructures of the World form from Superclusters down to Galaxies, Extrasolar systems, planets, and moons. Formation of galaxies and stars is not a process that concluded ages ago; instead, it is ongoing in the Luminous Epoch;

- Luminous Epoch spans from 0.4 billion years up to the present Epoch (13.8 billion years). The Big Bang discussed in the standard cosmological model is, in our view, the transition from Dark Epoch to Luminous Epoch.

A number of ideas presented in this paper are not new, and we don't claim credit for them. In fact, several ideas belonging to classical scientists such as Riemann, Maxwell, Heaviside, Planck, and Dirac are revisited in a new light. In the present manuscript we are attempting to describe the World while unifying and simplifying existing models and results into a cohesive physical picture of Dark Matter Cosmology and Astrophysics.

This manuscript concludes the series of papers on World-Universe Model published by "Journal of High Energy Physics, Gravitation and Cosmology" journal [1]-[12]. Many results obtained there are quoted in the current work without a full justification; an interested reader is encouraged to view the referenced papers in such cases. In this manuscript we discuss the proposed Multiworld and Dark Matter Fermi Bubbles for the first time.

In Chapter 2 we consider the origin, evolution, structure, ultimate fate, and primary cosmological parameters of the World. In Chapter 3 we discuss the main physical phenomena of Luminous Matter Astrophysics: Intergalactic Plasma, Microwave Background Radiation, Energy-Varying Photons, Mass-Varying Neutrinos, Cosmic Far-Infrared Background, and Time Delay of Fast Radio Bursts. Chapter 4 deals with the proposed Multicomponent DM, Macroobjects Cores made up of DMPs, and Weak Interaction providing integrity of all DM Cores. Chapter 5 discusses Dark Matter Cosmology including Dark Epoch, Rotational Fission, Luminous Epoch, and Distribution of the World's Energy Density. In Chapter 6 we explain different phenomena of DM Astrophysics: Multiwave- 
length Pulsars, Binary Millisecond Pulsars, Gamma-Ray Bursts, and Young Stellar Object Dippers in frames of the proposed Macroobject Shell Model. We also discuss the proposed Multiworld and Dark Matter Fermi Bubbles. Chapter 7 debates different physical phenomena of Solar System: Angular momentum, Gravitationally-Rounded Objects Internal Heat, Dark Matter Cores of Macroobjects, Evolution of the Sun, Solar Corona, Geocorona, Planetary Coronas, and High-Energy Atmospheric Physics including Ball Lightning.

\section{Cosmology}

WUM utilizes the following Basic ideas:

Hypersphere World as a model of a finite universe was proposed by Georg Riemann in 1854 [13]. WUM views the World as a 3-dimensional Hypersphere that expands along the fourth spatial dimension in the Universe. A Hypersphere is an example of a 3-Manifold which locally behaves like regular Euclidean 3-dimensional space, just as the surface of a sphere looks like a plane to small enough observers.

Principal Role of Maxwell's Equations (ME) that form the foundation of classical electrodynamics. Gravitoelectromagnetism (GEM) refers to a gravitational and electromagnetic analogy. The equations for GEM were first published by O. Heaviside in 1893 as a separate theory expanding Newton's law [14]. WUM follows this theory [5]. Maxwell's equations produce only two physically measurable quantities: energy density and energy flux density [9].

Existence of the Medium of the World stated by Nikola Tesla: "All attempts to explain the workings of the universe without recognizing the existence of the ether and the indispensable function it plays in the phenomena are futile and destined to oblivion" [15]. Paul Dirac stated in 1951 in an article in Nature, titled "Is there an Aether?" that "we are rather forced to have an aether" [16].

Variable Gravitational Parameter. The hypothesis was first proposed by Paul Dirac in 1937 [17].

Continuous Creation of Matter. In 1964 F. Hoyle and J. V. Narlikar explained the appearance of new matter by postulating the existence of what they dubbed the "creation field", or just the "C-field" [18]. P. Dirac in 1974 discussed the mechanisms of the additive and multiplicative creation of Matter [19].

Mach's Principle. A very general statement of Mach's principle is: "Local physical laws are determined by the large-scale structure of the universe".

Fundamental Parameters. Two Fundamental parameters in various rational exponents define all macro and micro features of the World: constant $\alpha$ (Sommerfeld's constant, see Section 2.5 for details) and dimensionless quantity $Q$, which increases in time, and is, in fact, a measure of the Size and Age of the World (see Section 2.6).

Key concepts and Observations of WUM are the following:

- Expansion and Creation of Matter;

- Content of the World; 
- Structure of Macroobjects;

- Inter-Connectivity of Primary Cosmological Parameters;

- Gravity, Space and Time are all emergent phenomena.

WUM makes reasonable assumptions in each of these areas. The remarkable agreement of the calculated values of the primary cosmological parameters with the observational data gives us considerable confidence in the Model.

\subsection{Expansion and Creation of Matter}

Before the Beginning of the World there was nothing but an Eternal Universe. About 14.2 billion years ago the World was started by a fluctuation in the Eternal Universe, and the Nucleus of the World, which is a four-dimensional ball, was born. An extrapolated Nucleus radius at the Beginning was equal to a basic unit of size $\alpha$ (see Section 2.5). 4-ball is the interior of a three-dimensional hypersphere. All points of the hypersphere are equivalent; there are no preferred centers or boundary of the World [5].

The 4-ball is expanding in the Eternal Universe, and its surface, the hypersphere, is likewise expanding. The radius of the Nucleus $R$ is increasing with speed $c$ (gravitoelectrodynamic constant) for the absolute cosmological time $\tau$ from the Beginning and equals to $R=c \tau$. The expansion of the Hypersphere World can be understood through the analogy with an expanding $3 \mathrm{D}$ balloon: imagine an ant residing on a seemingly two-dimensional surface of a balloon. As the balloon is blown up, its radius increases, and its surface grows. The distance between any two points on the surface increases. The ant sees her world expand but does not observe a preferred center.

According to WUM, the surface of the 4-ball is created in a process analogous to sublimation. Continuous creation of matter is the result of such process (see Section 2.8). Sublimation is a well-known endothermic process that happens when surfaces are intrinsically more energetically favorable than the bulk of a material, and hence there is a driving force for surfaces to be created. Matter arises from the fourth spatial dimension. The Universe is responsible for the creation of Matter. Dark Matter Particles (DMPs) carry new Matter into the World [5].

It is important to emphasize that:

- Creation of Matter is a direct consequence of expansion;

- Creation of Dark Matter (DM) occurs homogeneously in all points of the hypersphere World;

- Luminous Matter is a byproduct of DM annihilation. Consequently, the matter-antimatter asymmetry problem discussed in literature does not arise (since antimatter does not get created by DM annihilation).

\subsection{Content of the World}

The existence of the Medium is a principal point of WUM. It follows from the observations of Intergalactic Plasma; Cosmic Microwave Background Radiation 
(MBR); Far-Infrared Background Radiation (FIRB). Inter-galactic voids discussed by astronomers are in fact examples of the Medium in its purest. Cosmic MBR is part of the Medium; it then follows that the Medium is the absolute frame of reference. Relative to MBR rest frame, Milky Way galaxy and Sun are moving with the speed of 552 and $370 \mathrm{~km} / \mathrm{s}$ respectively [5].

Theory of a Rotationally Elastic Medium. Long time ago it was realized that there are no transverse waves in the Aether, and hence the Aether could not be an elastic matter of an ordinary type. In 1846 James McCullagh proposed a theory of a rotationally elastic medium, i.e. a medium in which every particle resists absolute rotation [20]. This theory produces equations analogous to ME. WUM is based on Maxwell's equations, and McCullagh's theory is a good fit for description of the Medium.

The Medium consists of stable elementary particles with lifetimes longer than the age of the World: protons, electrons, photons, neutrinos, and dark matter particles. For all particles under consideration we use the following characteristics:

- Type of particle (fermion or boson);

- "Mass" that is equivalent to "Rest energy" with the constant $c^{2}$;

- Electrical charge.

The total energy density of the Medium is $2 / 3$ of the overall energy density of the World (see Section 2.8). Superclusters, Galaxies, Extrasolar systems, planets, moons, etc. are made of the same particles. The energy density of Macroobjects adds up to $1 / 3$ of the total energy density of the World throughout the World's evolution [5].

\subsection{Structure of Macroobjects}

In our view, all Macroobjects (MOs) of the World (galaxies, extrasolar systems, planets, and moons) possess the following properties [8]:

- Macroobject nuclei are made up of self-annihilating DMFs;

- MOs contain other particles, including DM and baryonic matter, in shells surrounding their nuclei.

WUM predicts existence of 5 types of self-annihilating DMPs with masses of 1.3 TeV, 9.6 GeV, $70 \mathrm{MeV}, 340 \mathrm{keV}$, and $3.7 \mathrm{keV}$ (see Section 4.1). The signs of annihilation of these particles are found in the observed gamma-ray spectra which we connect with the structure of MOs (nuclei and shells composition). Annihilation of those DMPs can give rise to any combination of gamma-ray lines. Thus, the diversity of Very High Energy gamma-ray sources in the World has a clear explanation in frames of WUM [8].

\subsection{Nucleosynthesis. Large-Scale Structures. Ultimate Fate}

Nucleosynthesis of all luminous elements (including light elements) occurs inside of DM Cores of all Macroobjects during their evolution. The theory of Stellar nucleosynthesis is well developed, starting with the publication of a celebrated $\mathrm{B}^{2} \mathrm{FH}$ review paper [21]. With respect to WUM, this theory should be ex- 
panded to include annihilation of heavy DMFs in MOs' Cores (see Section 4.1). The amount of energy produced due to this process is sufficiently high to create all elements inside of MOs' [5].

Formation and Evolution of Large-Scale Structures. All Macroobjects of the World have Cores made up of different DMPs. The matter creation is occurring homogeneously in all points of the World. It follows that new stars can be created inside of galaxies, new galaxies can be created inside of superclusters, which can arise in the World. Structures form in parallel around different Cores made of different DMPs. Formation of galaxies and stars is not a process that concluded ages ago; instead, it is ongoing [5].

Ultimate Fate of the World. The Universe is continuously creating Matter in the World. Assuming an Eternal Universe, the numbers of cosmological structures and their size on all levels will increase. The temperature of the Medium will asymptotically reach zero [1].

\subsection{Fundamental Parameters and Basic Units}

It is the main goal of WUM to develop a Model based on two dimensionless Fundamental Parameters only: the constant $\alpha$ and the time-varying parameter $Q$, which is a measure of the Size and Age of the World. In WUM we often use well-known physical parameters, keeping in mind that all of them can be expressed through the Basic Units. Taking the relative values of physical parameters in terms of the Basic Units we can express all dimensionless parameters of the World through two Fundamental Parameters $\alpha$ and $Q$ in various rational exponents, as well as small integer numbers and $\pi$.

To define the values of the constant $\alpha$ and $a$ we analyze the history of the Classical Physics [10]:

- The electrodynamic constant $c$ in Maxwell's equations was measured by Weber and Kohlrausch in 1857 [22];

- Rydberg constant $R_{\infty}=\alpha^{3} / 2 a$ is a physical constant relating to atomic spectra. The constant first arose in 1888 as an empirical fitting parameter in the Rydberg formula for the hydrogen spectral series [23]. As of 2018, $R_{\infty}$ is the most accurately measured Fundamental constant;

- Electron Charge-to-Mass Ratio $e / m_{e}$ is a Quantity in experimental physics. It bears significance because the electron mass $m_{e}$ cannot be measured directly. The $e / m_{e}$ ratio of an electron was successfully calculated by J. J. Thomson in 1897 [24]. We define it after Thomson: $R_{T} \equiv e / m_{e}$;

- Planck constant $h$, which is generally associated with the behavior of microscopically small systems, was introduced and measured by Max Planck in 1901 based on statistical thermodynamic analysis of the black-body radiation [25];

- The magnetic constant: $\mu_{0}=4 \pi \times 10^{-7} \mathrm{H} / \mathrm{m}$.

Based on the experimentally measured values of the constants $c, R_{\infty}, R_{T}, h$ we calculate the most important Fundamental constants as follows: 


$$
\begin{gathered}
\alpha=\left[2\left(\mu_{0} h / c\right) R_{\infty}^{2} R_{T}^{2}\right]^{1 / 5} \\
a=\left[\frac{\left(\mu_{0} h / c\right)^{3} R_{\infty} R_{T}^{6}}{4}\right]^{1 / 5} \\
m_{e}=\frac{h}{c}\left[\frac{8 R_{\infty}}{\left(\mu_{0} h / c\right)^{2} R_{T}^{4}}\right]^{1 / 5} \\
e=\left(\frac{2 \alpha h / c}{\mu_{0}}\right)^{1 / 2}
\end{gathered}
$$

All these Fundamental constants, including classical electron radius $a_{o}=a / 2 \pi$, were measured and could be calculated before Quantum Mechanics. The calculated constant $a$ is the basic unit of size in WUM. It is worth to note that the constant $\alpha$ was later named "Sommerfeld's constant" and then "Fine-structure constant".

Below we will refer to the following Basic Units:

- energy $E_{0}=\frac{h c}{a}$;

- energy density $\rho_{0}=\frac{h c}{a^{4}}$;

- $\quad$ surface energy density $\sigma_{0}=\frac{h c}{a^{3}}$;

- mass $m_{0}=\frac{h}{a c}$;

- time $t_{0}=\frac{a}{c}$;

- frequency $v_{0}=\frac{c}{a}$.

\subsection{Inter-Connectivity of Primary Cosmological Parameters}

The constancy of the universe fundamental constants, including Newtonian constant of gravitation and Planck mass, is now commonly accepted, although has never been firmly established as a fact. All conclusions on the (almost) constancy of the Newtonian parameter of gravitation are model-dependent. A commonly held opinion states that gravity has no established relation to other fundamental forces, so it does not appear possible to calculate it from other constants that can be measured more accurately, as is done in some other areas of physics. WUM holds that there indeed exist relations between all primary cosmological parameters that depend on dimensionless time-varying quantity $Q$, which equals to: $Q=\tau / t_{0} \quad[4]$.

The model develops a mathematical framework that allows for direct calculation of the following primary cosmological parameters through $Q$ [7]:

- Newtonian parameter of gravitation $G$.

$$
G=\frac{a^{2} c^{4}}{8 \pi h c} \times Q^{-1}
$$


- Hubble's parameter $H$ :

$$
H=v_{0} \times Q^{-1}
$$

- Age of the World $A_{\tau}$ :

$$
A_{\tau}=t_{0} \times Q
$$

- The Worlds' radius of curvature in the fourth spatial dimension $R$ :

$$
R=a \times Q
$$

- Critical energy density $\rho_{c r}$ :

$$
\rho_{c r}=3 \rho_{0} \times Q^{-1}
$$

- Concentration of Intergalactic Plasma $n_{I G P}$ :

$$
n_{I G P}=\frac{2 \pi^{2}}{a^{3}} \frac{m_{e}}{m_{p}} \times Q^{-1}
$$

- Minimum Energy of Photons $E_{p h}$ :

$$
E_{p h}=\left(\frac{m_{e}}{m_{p}}\right)^{1 / 2} E_{0} \times Q^{-1 / 2}
$$

- Temperature of the Microwave Background Radiation $T_{M B R}$ :

$$
T_{M B R}=\frac{E_{0}}{k_{B}}\left(\frac{15 \alpha}{2 \pi^{3}} \frac{m_{e}}{m_{p}}\right)^{1 / 4} \times Q^{-1 / 4}
$$

- Temperature of the Far-Infrared Background Radiation Peak $T_{F I R B}$ :

$$
T_{F I R B}=\frac{E_{0}}{k_{B}}\left(\frac{15}{4 \pi^{5}}\right)^{1 / 4} \times Q^{-1 / 4}
$$

where $k_{B}$ is the Boltzmann constant that was measured by Planck in 1901 [25]; $m_{e} / m_{p}$ is electron-to-proton mass ratio.

In frames of WUM, we calculate the values of these parameters, which are in good agreement with the latest results of their measurements. For example, calculating the value of Hubble's parameter $H_{0}$ based on the average value of the gravitational parameter $G$ we find $H_{0}=68.7457 \mathrm{~km} / \mathrm{s} \cdot \mathrm{Mpc}$, which is in good agreement with $H_{0}=69.32 \pm 0.8 \mathrm{~km} / \mathrm{s} \cdot \mathrm{Mpc}$ obtained using WMAP data [26]. Note that the precision of $H_{0}$ value has increased by three orders of magnitude. Similar precision enhancement holds for other parameters' values as well.

The remarkable agreement of the calculated values of the primary cosmological parameters with the observational data (see Sections 3.1, 3.2, 3.3, and 3.5) gives us considerable confidence in the Model. We propose to introduce $Q$ as a new Fundamental Parameter tracked by CODATA and use its value in calculation of all $Q$-dependent parameters.

\subsection{Hypersphere World}

The physical laws we observe appear to be independent of the Worlds' curvature in the fourth spatial dimension due to the very small value of the dimen- 
sion-transposing gravitomagnetic parameter of the Medium [1]. Consequently, direct observation of the Worlds' curvature would appear to be a hopeless goal.

One way to prove the existence of the Worlds' curvature is direct measurement of truly large-scale parameters of the World: Gravitational, Hubble's, Temperature of the Microwave Background Radiation. Conducted at various points of time, these measurements would give us varying results, providing insight into the curved nature of the World. Unfortunately, the accuracy of the measurements is quite poor. Measurement errors far outweigh any possible "curvature effects", rendering this technique useless in practice. To be conclusive, the measurements would have to be conducted billions of years apart [5].

In WUM, Local Physics is linked with the large-scale structure of the Hypersphere World through the dimensionless quantity $Q$. The proposed approach to the fourth spatial dimension agrees with Mach's principle: “Local physical laws are determined by the large-scale structure of the universe". Applied to WUM, it follows that all parameters of the World depending on $Q$ are a manifestation of the Worlds' curvature in the fourth spatial dimension [5].

\subsection{Critical Energy Density}

The principal idea of WUM is that the energy density of the World $\rho_{W}$ equals to the critical energy density $\rho_{c r}$ necessary for 3-Manifold at any cosmological time. $\rho_{c r}$ can be found by considering a sphere of radius $R_{M}$ and enclosed mass $M$ that can be calculated by multiplication of critical density by the volume of the sphere. When the World has the critical density, the Hubble velocity $H \times R_{M}$ is equal to the escape velocity, which gives an equation for the mass $M$ leading to the equation for $\rho_{c r}$ [27]:

$$
\rho_{c r}=\frac{3 H^{2} c^{2}}{8 \pi G}
$$

This equation can be rewritten as [1]:

$$
\frac{4 \pi G}{c^{2}} \times \frac{2}{3} \rho_{c r}=\mu_{g} \times \rho_{M}=H^{2}=\frac{c^{2}}{R^{2}}
$$

where $\mu_{g}=\frac{4 \pi G}{c^{2}}$ is a gravitomagnetic parameter and $\rho_{M}=\frac{2}{3} \rho_{c r}$ is the energy density of the Medium.

According to WUM, creation of Matter in the Hypersphere World occurs continually through a process analogous to sublimation (see Section 2.1). The Eternal Universe is responsible for the creation of Matter. The physical conditions at the expanding 4-ball Nucleus of the World and Universe boundary remain constant in all times. If we assume that the content of Matter in 4-ball Nucleus is proportional to the surface of the 4-ball (hypersphere) and basic unit of surface energy density $\sigma_{0}$, then an energy density of the Nucleus $\rho_{N}$ :

$$
\rho_{N}=\frac{2 \pi^{2} R^{3} \sigma_{0}}{0.5 \pi^{2} R^{4}}=\frac{4 h c}{a^{3} R}=4 \rho_{0} \times Q^{-1}
$$


is higher than the critical energy density of the World: $\rho_{c r}=3 \rho_{0} \times Q^{-1}$. It means that the surface of the 4-ball Nucleus is intrinsically more energetically favorable than the bulk and hence there is a driving force for surface to be created. It is worth to note that energy density of the Nucleus $\rho_{N} \propto R^{-1}$, and hence the surface energy density of the hypersphere $\rho_{c r} \propto R^{-1}$. Considering that $H \propto R^{-1}$, it is easy to see that the gravitational parameter $G \propto R^{-1}$ [1].

\subsection{Gravity, Space and Time}

In frames of WUM, the parameter $G$ can be calculated based on the value of the energy density of the Medium $\rho_{M}$ of the World [1]:

$$
G=\frac{\rho_{M}}{4 \pi} \times P^{2}
$$

where a dimension-transposing parameter $P$ equals to:

$$
P=\frac{a^{3} c^{2}}{2 h c}
$$

Then the Newton's law of universal gravitation can be rewritten in the following way:

$$
F=G \frac{m \times M}{r^{2}}=\frac{\rho_{M}}{4 \pi} \frac{\frac{a^{3}}{2 L_{C m}} \times \frac{a^{3}}{2 L_{C M}}}{r^{2}}
$$

where we introduced the measurable parameter of the Medium $\rho_{M}$ instead of the phenomenological coefficient $G$; and gravitoelectromagnetic charges $\frac{a^{3}}{2 L_{C m}}$ and $\frac{a^{3}}{2 L_{C M}}$ instead of macroobjects masses $m$ and $M\left(L_{C m}\right.$ and $L_{C M}$ are Compton length of mass $m$ and $M$ respectively). The gravitoelectromagnetic charges have a dimension of "Area", which is equivalent to "Energy", with the constant that equals to the basic unit of surface energy density $\sigma_{0}$.

Following WUM approach, we can find a gravitomagnetic parameter of the Medium $\mu_{M}$ [1]:

$$
\mu_{M}=R^{-1}
$$

and the impedance of the Medium $Z_{M}$ :

$$
Z_{M}=\mu_{M} c=H=\tau^{-1}
$$

These parameters are analogous to the magnetic constant $\mu_{0}$ and impedance of electromagnetic field $Z_{0}=\mu_{0} c$.

It follows that measuring the value of Hubble's parameter anywhere in the World and taking its inverse value allows us to calculate the absolute Age of the World. The Hubble's parameter is then the most important characteristic of the World, as it defines the Worlds' Age. While in our Model Hubble's parameter $H$ has a clear physical meaning, the gravitational parameter $G=\frac{a^{3} c^{3}}{8 \pi h c} H$ is a 
phenomenological coefficient in the Newton's law of universal gravitation.

The second important characteristic of the World is the gravitomagnetic parameter $\mu_{M}$. Taking its inverse value, we can find the absolute radius of curvature of the World in the fourth spatial dimension. We emphasize that the above two parameters $\left(Z_{M}\right.$ and $\mu_{M}$ ) are principally different physical characteristics of the Medium that are connected through the gravitoelectrodynamic constant $c$. It means that Time is not a physical dimension and is absolutely different entity than Space. Time is a factor of the World.

In WUM, Time and Space are closely connected with Mediums' impedance and gravitomagnetic parameter. It follows that neither Time nor Space could be discussed in absence of the Medium. The gravitational parameter $G$ that is proportional to the Mediums' energy density can be introduced only for the Medium filled with Matter. In frames of WUM, the Gravitation is a result of simple interactions of Dark Matter Fermions Dions with Matter that work cooperatively to create a more complex interaction. Dions are responsible for the Le Sage's mechanism of the gravitation [4].

As the conclusion, Gravity, Space and Time are all emergent phenomena [5]. In this regard, it is worth to recall the Albert Einstein quote: "When forced to summarize the theory of relativity in one sentence: time and space and gravitation have no separate existence from matter".

\section{Luminous Matter Astrophysics}

\subsection{Intergalactic Plasma}

In our Model, the World consists of stable elementary particles with lifetimes longer than the age of the World. Protons with mass $m_{p}$ and electrons with mass $m_{e}$ have identical concentrations in the World: $n_{p}=n_{e}$. Low density intergalactic plasma consisting of protons and electrons has the lowest plasma frequency $\omega_{p l}[1]$ :

$$
\omega_{p l}^{2}=\frac{4 \pi n_{p} e^{2}}{4 \pi \varepsilon_{0} m_{p}}=\frac{m_{e}}{m_{p}} \frac{4 \pi n_{e} e^{2}}{4 \pi \varepsilon_{0} m_{e}}=\frac{m_{e}}{m_{p}} \omega_{e}^{2}
$$

where $e$ is the elementary charge, $\varepsilon_{0}$ is the electric constant, and $\omega_{e}$ is electron plasma frequency. If we assume that $\omega_{e}$ is proportional to $Q^{-1 / 2}$, then $n_{e}$ is proportional to $Q^{-1}$. Energy densities of protons and electrons are then proportional to $Q^{-1}$, similar to the critical energy density $\rho_{c r} \propto Q^{-1}$. Considering $\omega_{e}=2 \pi v_{0} \times Q^{-1 / 2}$, we can calculate concentration of protons and electrons:

$$
n_{p}=n_{e}=\frac{2 \pi^{2}}{a^{3}} \frac{m_{e}}{m_{p}} \times Q^{-1}=0.25480 \mathrm{~m}^{-3}
$$

A. Mirizzi, et al. found that the mean diffuse intergalactic plasma density is bounded by $n_{e} \lesssim 0.27 \mathrm{~m}^{-3}$ [28]. The calculated Mediums' plasma density is in good agreement with the estimated value [28].

$\rho_{p}=n_{p} m_{p} c^{2}$ is the energy density of protons in the Medium. The relative energy density of protons $\Omega_{p}$ is then the ratio of $\rho_{p} / \rho_{c r}$ : 


$$
\Omega_{p}=\frac{\rho_{p}}{\rho_{c r}}=\frac{2 \pi^{2} \alpha}{3}=0.048014655
$$

This value is in good agreement with experimentally found value of $0.049 \pm$ 0.013 [29]. It is worth to note that the relative energy density of protons in Luminous Epoch is constant all time and proportional to the Fundamental constant $\alpha$.

\subsection{Microwave Background Radiation}

According to WUM, the black body spectrum of MBR is due to thermodynamic equilibrium of photons with low density intergalactic plasma consisting of protons and electrons. $\rho_{e}=n_{e} m_{e} c^{2}$ is the energy density of electrons in the Medium. We assume that the energy density of MBR $\rho_{M B R}$ equals to twice the value of $\rho_{e}$ (considering two polarizations of photons) [1]:

$$
\rho_{M B R}=2 \rho_{e}=4 \pi^{2} \alpha \frac{m_{e}}{m_{p}} \rho_{0} \times Q^{-1}=\frac{8 \pi^{5}}{15} \frac{k_{B}^{4}}{(h c)^{3}} T_{M B R}^{4}
$$

where $T_{M B R}$ is MBR temperature. We can now calculate the value of $T_{M B R}$ :

$$
T_{M B R}=\frac{E_{0}}{k_{B}}\left(\frac{15 \alpha}{2 \pi^{3}} \frac{m_{e}}{m_{p}}\right)^{1 / 4} \times Q^{-1 / 4}=2.72518 \mathrm{~K}
$$

which is in excellent agreement with experimentally measured value of 2.72548 $\pm 0.00057 \mathrm{~K}$ [30]. We are not aware of any other model that allows calculation of MBR temperature with such accuracy.

\subsection{Energy-Varying Photons}

From Equation (3.1.1) we obtain the value of the lowest frequency $v_{p l}[1]$ :

$$
v_{p l}=\frac{\omega_{p l}}{2 \pi}=\left(\frac{m_{e}}{m_{p}}\right)^{1 / 2} v_{0} \times Q^{-1 / 2}=4.5322 \mathrm{~Hz}
$$

Photons with energy smaller than $E_{p h}=h v_{p l}$ cannot propagate in plasma, thus $h v_{p l}$ is the smallest amount of energy a photon may possess. Following L. Bonetti, et al. [31] we can call this amount of energy the rest energy of photons that equals to

$$
E_{p h}=\left(\frac{m_{e}}{m_{p}}\right)^{1 / 2} E_{0} \times Q^{-1 / 2}=1.8743 \times 10^{-14} \mathrm{eV}
$$

The above value is in good agreement with the value $E_{p h} \lesssim 2.2 \times 10^{-14} \mathrm{eV}$ estimated by L. Bonetti, et al. [31]. It is more relevant to call $E_{p h}$ the minimum energy of photons which can pass through the Intergalactic plasma. It is worth to note that $E_{p h}$ is varying in time: $E_{p h} \propto \tau^{-1 / 2}$.

\subsection{Mass-Varying Neutrinos}

It is now established that there are three different types of neutrino: electronic $v_{e}$, muonic $v_{\mu}$, and tauonic $v_{\tau}$. Neutrino oscillations imply that neutrinos 
have non-zero masses. Let's take neutrino masses $m_{v_{e}}, m_{v_{\mu}}, m_{v_{\tau}}$ that are near [3]:

$$
m_{v}=m_{0} \times Q^{-1 / 4}
$$

Their concentrations $n_{v}$ are then proportional to

$$
n_{v} \propto \frac{1}{a^{3}} \times Q^{-3 / 4}
$$

and their energy density $\rho_{v}$ is then proportional to $Q^{-1}$, similar to critical energy density $\rho_{c r} \propto Q^{-1}$.

Experimental results obtained by M. Sanchez [32] show $v_{e} \rightarrow v_{\mu, \tau}$ neutrino oscillations with parameter $\Delta m_{\text {sol }}^{2}$ given by

$$
2.3 \times 10^{-5} \mathrm{eV}^{2} / c^{4} \leq \Delta m_{\text {sol }}^{2} \leq 9.3 \times 10^{-5} \mathrm{eV}^{2} / c^{4}
$$

and $v_{\mu} \rightarrow v_{\tau}$ neutrino oscillations with parameter $\Delta m_{a t m}^{2}$ :

$$
1.6 \times 10^{-3} \mathrm{eV}^{2} / c^{4} \leq \Delta m_{\text {atm }}^{2} \leq 3.9 \times 10^{-3} \mathrm{eV}^{2} / c^{4}
$$

where $\Delta m_{\text {sol }}^{2}$ and $\Delta m_{\text {atm }}^{2}$ are mass splitting for solar and atmospheric neutrinos respectively. Significantly more accurate result was obtained by P. Kaus, et al. [33] for the ratio of the mass splitting:

$$
\sqrt{\frac{\Delta m_{s o l}^{2}}{\Delta m_{\text {atm }}^{2}}} \cong 0.16 \cong \frac{1}{6}
$$

Let's assume that muonic neutrino's mass indeed equals to

$$
m_{v_{\mu}}=m_{v}=m_{0} \times Q^{-1 / 4} \cong 7.5 \times 10^{-3} \mathrm{eV} / c^{2}
$$

From Equation (3.4) it then follows that

$$
m_{v_{\tau}}=6 m_{v} \cong 4.5 \times 10^{-2} \mathrm{eV} / c^{2}
$$

Then the squared values of the muonic and tauonic neutrino masses fall into the experimentally found ranges:

$$
\begin{aligned}
& m_{v_{\mu}}^{2} \cong 5.6 \times 10^{-5} \mathrm{eV}^{2} / c^{4} \\
& m_{v_{\tau}}^{2} \cong 2 \times 10^{-3} \mathrm{eV}^{2} / c^{4}
\end{aligned}
$$

We assume that electronic neutrino mass equals to [3]:

$$
m_{v_{e}}=\frac{1}{24} m_{v} \cong 3.1 \times 10^{-4} \mathrm{eV} / c^{2}
$$

The sum of the calculated neutrino masses: $\Sigma m_{v} \cong 0.053 \mathrm{eV} / c^{2}$ is in good agreement with the value of $0.06 \mathrm{eV} / c^{2}$ discussed in literature [34].

\subsection{Cosmic Far-Infrared Background}

The cosmic Far-Infrared Background (FIRB), which was announced in 1998, is part of the Cosmic Infrared Background, with wavelengths near 100 microns that is the peak power wavelength of the black-body radiation at temperature 29 K. According to WUM, large cosmic grains are responsible for the FIRB [3]. 
It was experimentally found that the size of large grains $D_{G}$ is roughly equal to the length $L_{F}=a \times Q^{1 / 4}$, and their mass $M_{G}$ is close to the Planck mass: $M_{P}=2 m_{0} \times Q^{1 / 2}$ [35] [36] [37]. A grain of mass $B_{1} M_{P}$ and radius $B_{2} L_{F}$ is receiving energy at the following rate:

$$
\frac{\mathrm{d}}{\mathrm{d} \tau}\left(B_{1} M_{P} c^{2}\right)=\frac{B_{1} M_{P} c^{2}}{2 \tau}
$$

where $B_{1}$ and $B_{2}$ are parameters. The received energy will increase the grain's temperature $T_{G}$, until equilibrium is achieved: power received equals to the power irradiated by the surface of a grain in accordance with the Stefan-Boltzmann law

$$
\frac{B_{1} M_{P} c^{2}}{2 \tau}=\sigma_{S B} T_{G}^{4} \times 4 \pi B_{2}^{2} L_{F}^{2}
$$

where $\sigma_{S B}$ is Stefan-Boltzmann constant: $\sigma_{S B}=\frac{2 \pi^{5} k_{B}^{4}}{15 h^{3} c^{3}}$. With Nikola Tesla's principle at heart-There is no energy in matter other than that received from the environment-we get:

$$
B_{1} M_{P} c^{2}=4 \pi B_{2}^{2} L_{F}^{2} \sigma_{0}
$$

We then calculate the grain's stationary temperature $T_{G}$ to be [3]:

$$
T_{G}=\left(\frac{15}{4 \pi^{5}}\right)^{1 / 4} \frac{h c}{k_{B} L_{F}}=28.955 \mathrm{~K}
$$

This result is in an excellent agreement with experimentally measured value of $29 \mathrm{~K}$ [38]-[49]. The total flux of the FIRB radiation is the sum of the contributions of all individual grains. Comparing Equations (3.2) and (3.5), we can find the relation between the grains' temperature and the temperature of the MBR:

$$
T_{G}=\left(3 \Omega_{e}\right)^{-1 / 4} \times T_{M B R}
$$

where electron relative energy density $\Omega_{e}$ equals to $\Omega_{e}=\frac{m_{e}}{m_{p}} \Omega_{p}$.

\subsection{Time Delay of Fast Radio Bursts}

Fast Radio Burst (FRB) is a high-energy astrophysical phenomenon manifested as a transient radio pulse lasting only a few milliseconds. The component frequencies of each burst are delayed by different amounts of time depending on the wavelength. This delay is described by a value referred to as a Dispersion Measure which is the total column density of free electrons between the observer and the source of FRB. Fast radio bursts have Dispersion measures which are consistent with propagation through ionized plasma [29].

Consider a photon with initial frequency $v_{\text {emit }}$ and energy $E_{\text {emit }}$ emitted at time $\tau_{e m i t}$ when the radius of the hypersphere World in the fourth spatial dimension was $R_{e m i t}$. The photon is continuously losing kinetic energy on its way to Earth until time $\tau_{o b s v}$ when the radius is $R_{o b s v}=R_{0}$. An observer will meas- 
ure $v_{\text {obsv }}$ and energy $E_{\text {obsv }}$ and calculate a redshift $z$. Recall that $\tau_{\text {emit }}$ and $\tau_{\text {obsv }}$ are cosmological times (ages of the World at the moments of emitting and observing).

A light-travel time distance to the source of FRB $d_{L T T}$ equals to [7]:

$$
d_{L T T}=c\left(\tau_{\text {obsv }}-\tau_{\text {emit }}\right)=c t_{L T T}=R_{0}-R_{\text {emit }}
$$

Let's calculate photons' traveling time $t_{p h}$ considering that the minimum energy of photons $E_{p h}$ is much smaller than the energy of photons $E_{\gamma}$ :

$$
t_{p h}=\frac{1}{c} \int_{R_{\text {emit }}}^{R_{0}}\left(1-\frac{E_{p h}^{2}}{E_{\gamma}^{2}}\right)^{-1 / 2} \mathrm{~d} r=t_{L T T}+\Delta t_{p h}
$$

where $\Delta t_{p h}$ is photons' time delay relative to the light-travel time $t_{L T T}$ that equals to

$$
\Delta t_{p h}=\frac{1}{2 c} \int_{R_{e m i t}}^{R_{0}} \frac{E_{p h}^{2}}{E_{\gamma}^{2}} \mathrm{~d} r
$$

All observed FRBs have redshifts $z<1$. It means that we can use the Hubble's law: $d_{L T T}=R_{0} z$. Then

$$
R_{\text {emit }}=(1-z) R_{0}
$$

Photons' minimum energy squared at radius $r$ between $R_{\text {emit }}$ and $R_{0}$ equals to (see Equation (3.3)):

$$
E_{p h}^{2}=\frac{m_{e}}{m_{p}} \frac{a}{r} E_{0}^{2}
$$

According to WUM, photons' energy $E_{\gamma}$ on the way to the observer can be expressed by the following equation [6]:

$$
E_{\gamma}=z E_{o b s v}+(1-z) \frac{R_{0}}{r} E_{o b s v}
$$

which reduces to $E_{\text {emit }}$ at (3.6.2) and to $E_{\text {obsv }}$ at $r=R_{0}$. Placing the values of the parameters (3.6.2), (3.6.3), (3.6.4) into (3.6.1), we have for photons' time delay [6]:

$$
\Delta t_{p h}=\frac{4.61}{z^{2}}\left[\ln \left(\frac{1}{1-z^{2}}\right)-\frac{z^{2}}{1+z}\right] \times\left(\frac{v}{1 \mathrm{GHz}}\right)^{-2}
$$

Taking $z=0.492$ [29] we get the calculated value of photons' time delay

$$
\Delta t_{p h}^{c a l}=2.189 \times\left(\frac{v}{1 \mathrm{GHz}}\right)^{-2}
$$

which is in good agreement with experimentally measured value [29]:

$$
\Delta t_{p h}^{e x p}=2.438 \times\left(\frac{v}{1 \mathrm{GHz}}\right)^{-2}
$$

It is worth to note that in our calculations there is no need in the dispersion measure, and time delay depends on the redshift only. 


\section{Dark Matter}

\subsection{Multicomponent Dark Matter}

DMPs might be observed in Centers of Macroobjects has drawn many new researchers to the field in the last forty years. Indirect effects in cosmic rays and gamma-ray background from the annihilation of cold DM in the form of heavy stable neutral leptons in Galaxies were considered in pioneer articles [50]-[55]. A mechanism whereby DM in protostellar halos plays the role in the formation of the first stars is discussed by D. Spolyar, K. Freese, and P. Gondolo [56]. Heat from neutralino DM annihilation is shown to overwhelm any cooling mechanism, consequently impeding the star formation process. A "dark star" powered by DM annihilation instead of nuclear fusion may result. Dark stars are in hydrostatic and thermal equilibrium, but with an unusual power source. Weakly Interacting Massive Particles (WIMPs) are among the best candidates for DM [57]. Important cosmological problems like Dark Matter and Dark Energy could be, in principle, solved through extended gravity. This is stressed, for example, in the famous paper of Prof. C. Corda [58].

Two-component DM system consisting of bosonic and fermionic components is proposed for the explanation of emission lines from the bulge of Milky Way galaxy. C. Boehm, P. Fayet, and J. Silk analyze the possibility of two coannihilating neutral and stable DMPs: a heavy fermion for example, like the lightest neutralino (>100 GeV) and the other one a possibly light spin-0 particle ( $100 \mathrm{MeV})$ [59].

WUM proposes multicomponent DM system consisting of two couples of coannihilating DMPs: a heavy DM fermion-DMF1 (1.3 TeV) and a light spin-0 boson-DIRAC $(70 \mathrm{MeV})$ that is a dipole of Dirac's monopoles; a heavy fermion-DMF2 $(9.6 \mathrm{GeV})$ and a light spin-0 boson-ELOP $(340 \mathrm{keV})$ that is a dipole of preons with electrical charge e/3; a self-annihilating fermion-DMF3 (3.7 keV) and a fermion DMF4 named Dion $(0.2 \mathrm{eV})$.

WUM postulates that masses of DMFs and bosons are proportional to $m_{0}$ multiplied by different exponents of $\alpha$ and can be expressed with the following formulae [11]:

DMF1 (fermion): $m_{D M F 1}=\alpha^{-2} m_{0}=1.3149950 \mathrm{TeV}$

DMF2 (fermion): $m_{D M F 2}=\alpha^{-1} m_{0}=9.5959823 \mathrm{GeV}$

DIRAC (boson): $m_{\text {DIRAC }}=\alpha^{0} m_{0}=70.025267 \mathrm{MeV}$

ELOP (boson): $m_{E L O P}=2 / 3 \alpha^{1} m_{0}=340.66606 \mathrm{keV}$

DMF3 (fermion): $m_{D M F 3}=\alpha^{2} m_{0}=3.7289402 \mathrm{keV}$

DMF4 (fermion): $m_{D M F 4}=\alpha^{4} m_{0}=0.19857111 \mathrm{eV}$

The values of mass of DMF1, DMF2, DMF3 fall into the ranges estimated in literature for neutralinos, WIMPs, and sterile neutrinos respectively [2]. DMF1, DMF2 and DMF3 partake in the self-annihilation interaction with strength equals to $\alpha^{-2}, \alpha^{-1}$ and $\alpha^{2}$ respectively.

\subsection{Macroobjects Cores Made up of Dark Matter Particles}

According to WUM, Macrostructures of the World (Superclusters, Galaxies, 
Extrasolar Systems) have Nuclei made up of DMFs, which are surrounded by Shells composed of DM and baryonic matter. The shells envelope one another, like a Russian doll. The lighter a particle, the greater the radius and the mass of its shell. Innermost shells are the smallest and are made up of heaviest particles; outer shells are larger and consist of lighter particles [11]. Table 1 describes the parameters of Macroobjects Cores (which are Fermionic Compact Stars in WUM) in the present Epoch made up of different DM fermions: self-annihilating DMF1, DMF2, DMF3 and the fermion DMF4 named Dion.

The calculated parameters of the shells show that [11]:

- Nuclei made of self-annihilating DMF1 and/or DMF2 compose Cores of stars in extrasolar systems;

- Shells of DMF3 around Nuclei made up of self-annihilating DMF1 and/or DMF2 make up Cores of galaxies;

- Nuclei made of DMF1 and/or DMF2 surrounded by shells of DMF3 and DMF4 compose Cores of superclusters.

Macroobjects Cores have the following properties [2]:

- The minimum radius of Core $R_{\min }$ made of any fermion equals to three Schwarzschild radii;

- Core density does not depend on $M_{\max }$ and $R_{\min }$ and does not change in time while $M_{\max } \propto \tau^{3 / 2}$ and $R_{\min } \propto \tau^{1 / 2}$.

K. Mehrgan, et al. observed a supergiant elliptical galaxy Holmberg 15A about 700 million light-years from Earth. They found an extreme core with a mass of 4 $\times 10^{10}$ solar masses at the center of Holm 15A [60]. The calculated maximum mass of galaxy Core of $6 \times 10^{10}$ solar masses (see Table 1 ) is in good agreement with the experimentally found value [60].

\subsection{Weak Interaction}

The widely discussed models for nonbaryonic DM are based on the Cold DM hypothesis, and corresponding particles are commonly assumed to be WIMPs, which interact via gravity and any other force (or forces), potentially not part of the standard model itself, which is as weak as or weaker than the weak nuclear force, but also non-vanishing in its strength (Wikipedia. Weakly interacting massive particles). It follows that a new weak force needs to exist, providing interaction

Table 1. Parameters of macroobjects cores made up of different DMFs in the present Epoch.

\begin{tabular}{ccccc}
\hline Fermion & $\begin{array}{c}\text { Fermion } \\
\text { mass } \\
\boldsymbol{m}_{\boldsymbol{f}} \mathrm{MeV}\end{array}$ & $\begin{array}{c}\text { Macroobject } \\
\text { mass } \\
M_{\max }, \mathrm{kg}\end{array}$ & $\begin{array}{c}\text { Macroobject } \\
\text { radius } \\
\boldsymbol{R}_{\min }, \mathrm{m}\end{array}$ & $\begin{array}{c}\text { Macroobject } \\
\text { density } \\
\rho_{\text {max }}, \mathrm{kg} / \mathrm{m}^{3}\end{array}$ \\
\hline DMF1 & $1.3 \times 10^{6}$ & $1.9 \times 10^{30}$ & $8.6 \times 10^{3}$ & $7.2 \times 10^{17}$ \\
DMF2 & $9.6 \times 10^{3}$ & $1.9 \times 10^{30}$ & $8.6 \times 10^{3}$ & $7.2 \times 10^{17}$ \\
DMF3 & $3.7 \times 10^{-3}$ & $1.2 \times 10^{41}$ & $5.4 \times 10^{14}$ & $1.8 \times 10^{-4}$ \\
DMF4 & $2 \times 10^{-7}$ & $4.2 \times 10^{49}$ & $1.9 \times 10^{23}$ & $1.5 \times 10^{-21}$ \\
\hline
\end{tabular}


between DMPs. The strength of this force exceeds that of gravity, and its range is considerably greater than that of the weak nuclear force.

According to WUM, strength of gravity is characterized by gravitational parameter [11]:

$$
G=G_{0} \times Q^{-1}
$$

where $G_{0}=\frac{a^{2} c^{4}}{8 \pi h c}$ is an extrapolated value of $G$ at the Beginning of the World $(Q=1) . Q$ in the present Epoch equals to [5]:

$$
Q=0.759972 \times 10^{40}
$$

The range of the gravity equals to the size of the World $R$ :

$$
R=a \times Q=1.34558 \times 10^{26} \mathrm{~m}
$$

In WUM, weak interaction is characterized by the parameter $G_{W}$ :

$$
G_{W}=G_{0} \times Q^{-1 / 4}
$$

which is about 30 orders of magnitude greater than $G$. The range of the weak interaction $R_{W}$ in the present Epoch equals to:

$$
R_{W}=a \times Q^{1 / 4}=1.65314 \times 10^{-4} \mathrm{~m}
$$

that is much greater than the range of the weak nuclear force. Calculated concentration of Dions $n_{D}$ in the largest shell of Superclusters: $n_{D} \cong 4.2 \times 10^{15} \mathrm{~m}^{-3}$ shows that a distance between particles is around $\sim 10^{-5} \mathrm{~m}$, which is much smaller than $R_{W}$. Thus, the introduced weak interaction between DMPs will provide integrity of all DM shells. In our view, weak interaction between particles DMF3 provides integrity of Fermi Bubbles (see Section 6.7).

\section{Dark Matter Cosmology}

\subsection{Dark Epoch}

Dark Epoch started at the Beginning of the World and lasted for about 0.4 billion years. WUM is a classical model, therefore classical notions can be introduced only when the very first ensemble of particles was created at the cosmological time $\cong 10^{-18} \mathrm{~s}$. At time $\tau \gg 10^{-18} \mathrm{~s}$ density fluctuations could happen in the Medium of the World filled with DMF1, DMF2, DIRACs, ELOPs, DMF3 and DMF4. The heaviest Dark Matter particles DMF1 could collect into a cloud with distances between particles smaller than $R_{W}$. As the result of the weak interaction, clumps of DMF1 will arise. Larger clumps will attract smaller clumps and DMPs and initiate a process of expanding the DM clump followed by growth of surrounding shells made up of other DMPs, up to the maximum mass of the shell made up of Dions at the end of Dark Epoch (0.4 billion years).

The process described above is the formation of the DM Core of a Supercluster [11]. We estimate the number of Supercluster Cores at present Epoch to be around $\sim 10^{3}$. DMPs supply not only additional mass $\left(\propto \tau^{3 / 2}\right)$ to Cores, but also additional angular momentum $\left(\propto \tau^{2}\right)$ fueling the overspinning of Dark Matter Cores 
(see next Section). In our opinion, all Supercluster Cores had undergone rotational fission at approximately the same cosmological time [11].

\subsection{Rotational Fission}

According to WUM, the rotational angular momentum of overspinning objects before rotational fission equals to [11]:

$$
L_{r o t}=\frac{4 \sqrt{2}}{15} \frac{1+5 \delta}{1+3 \delta} G^{0.5} M^{1.5} R^{0.5}
$$

where $M$ is a mass of overspinning object, $R$ is its radius, $\delta$ is the density ratio inside of the object: $\delta=\rho_{\min } / \rho_{\max }$. Parameters $G, M, R$ for Macroobjects Cores are time-varying: $G \propto \tau^{-1}, M \propto \tau^{3 / 2}$ and $R \propto \tau^{1 / 2}$. It follows that the rotational angular momentum of Cores $L_{\text {rot }}$ is proportional to $\tau^{2}$.

Let's introduce Age parameter $\theta_{F}$ that is a ratio of cosmological time of Core fission $\tau_{F}$ to the age of the World in present Epoch $A_{W}: \theta_{F}=\tau_{F} / A_{W}$. Finally, for $L_{r o t}$ at the time of Core fission we obtain the following equation [11]:

$$
L_{r o t}=\frac{4 \sqrt{2}}{15} \frac{1+5 \delta}{1+3 \delta} G^{0.5} M^{1.5} R^{0.5} \theta_{F}^{2}
$$

where for parameters $G, M, R$ we use their values in the present Epoch.

Local Supercluster (LS) is a mass concentration of galaxies containing the Local Group, which in turn contains the Milky Way galaxy. At least 100 galaxy groups and clusters are located within its diameter of 110 million light-years. Considering parameters of Dions' shell (see Table 1), we calculate the rotational angular momentum $L_{r o t}^{L S C}$ of LS Core before rotational fission with the age parameter $\theta_{0.4} \cong 1 / 36$ :

$$
L_{r o t}^{L S C}=3.7 \times 10^{77} \mathrm{~J} \cdot \mathrm{S}
$$

Milky Way (MW) is gravitationally bounded with LS [61]. Let's compare $L_{r o t}^{L S C}$ with an orbital momentum of Milky Way $L_{o r b}^{M W}$ calculated based on the distance of 65 million light years from LS Core and orbital speed of about 400 $\mathrm{km} / \mathrm{s}[61]$ :

$$
L_{o r b}^{M W}=2.5 \times 10^{71} \mathrm{~J} \cdot \mathrm{S}
$$

It means that as the result of rotational fission of LS Core, approximately $\sim 10^{6}$ galaxies like Milky Way could be generated at the same time. Considering that density of galaxies in the LS falls off with the square of the distance from its center near the Virgo Cluster, and the location of MW on the outskirts of the LS [62], the actual number of created galaxies could be much larger.

The mass-to-light ratio of the LS is about 300 times larger than that of the Solar ratio. Similar ratios are obtained for other superclusters [63]. These facts support the rotational fission mechanism proposed above. In 1933, Fritz Zwicky investigated the velocity dispersion of Coma cluster and found a surprisingly high mass-to-light ratio ( 500). He concluded: if this would be confirmed, we would get the surprising result that dark matter is present in much greater amount than 
luminous matter [64]. These ratios are one of the main arguments in favor of presence of large amounts of Dark Matter in the World.

Analogous calculations for MW Core based on parameters of DMF3 shell (see Table 1) produce the following value of rotational angular momentum $L_{r o t}^{M W C}$ [11]:

$$
L_{\text {rot }}^{M W C}=2.4 \times 10^{60} \mathrm{~J} \cdot \mathrm{s}
$$

which far exceeds the orbital momentum of the Solar System $L_{\text {orb }}^{S S}$ calculated based on the distance from the galactic center of 26,400 light years and orbital speed of about $220 \mathrm{~km} / \mathrm{s}$ :

$$
L_{\text {orb }}^{S S}=1.1 \times 10^{56} \mathrm{~J} \cdot \mathrm{S}
$$

As the result of rotational fission of MW Core 13.8 billion years ago, approximately $\sim 10^{4}$ Extrasolar systems like Solar System could be created at the same time. Considering that MW has grown inside out (in the present Epoch, most old stars can be found in the middle, more recently formed ones on the outskirts [65]), the number of generated Extrasolar systems could be much larger. Extrasolar system Cores can give birth to planetary cores, which in turn can generate cores of moons by the same Rotational Fission mechanism (see Section 7.1).

The oldest known star HD 140283 (Methuselah star) is a subgiant star about 190 light years away from Earth for which a reliable age has been determined [66]. H. E. Bond, et al. found its age to be $14.46+/-0.8 \mathrm{Gyr}$ that does not conflict with the age of the Universe, $13.77+/-0.06 \mathrm{Gyr}$, based on the microwave background and Hubble constant [66]. It means that this star must have formed between 13.66 and $13.83 \mathrm{Gyr}$, amount of time that is too short for formation of second generation of stars according to prevailing theories. In our Model this discovery can be explained by generation of HD 140283 by overspinning Core of the MW 13.8 billion years ago.

In frames of the developed Rotational Fission model it is easy to explain hyper-runaway stars unbound from the Milky Way with speeds of up to $\sim 700 \mathrm{~km} / \mathrm{s}$ [67]: they were launched by overspinning Core of the Large Magellan Cloud with the speed higher than the escape velocity [11].

\subsection{Luminous Epoch}

Luminous Epoch spans from 0.4 billion years up to the present Epoch (during 13.8 billion years). According to WUM, Cores of all Macroobjects (MOs) of the World (Superclusters, Galaxies, Extrasolar systems) possess the following properties [11]:

- Their Nuclei are made up of DMFs and contain other particles, including Dark Matter and baryonic matter, in shells surrounding the Nuclei;

- DMPs are continuously absorbed by Cores of all MOs. Luminous Matter (about 7.2\% of the total Matter in the World) is a byproduct of DMPs annihilation. Luminous Matter is re-emitted by Cores of MOs continuously;

- Nuclei and shells are growing in time: size $\propto \tau^{1 / 2}$; mass $\propto \tau^{3 / 2}$; and rota- 
tional angular momentum $\propto \tau^{2}$, until they reach the critical point of their stability, at which they detonate. Satellite cores and their orbital $L_{o r b}$ and rotational $L_{r o t}$ angular momenta released during detonation are produced by Overspinning Core (OC). The detonation process does not destroy OC; it's rather gravitational hyper-flares;

- Size, mass, composition, $L_{o r b}$ and $L_{r o t}$ of satellite cores depend on local density fluctuations at the edge of OC and cohesion of the outer shell. Consequently, the diversity of satellite cores has a clear explanation.

WUM refers to OC detonation process as Gravitational Burst (GB), analogous to Gamma Ray Burst [6]. In frames of WUM, the repeating GBs can be explained the following way:

- As the result of GB, the OC loses a small fraction of its mass and a large part of its rotational angular momentum;

- After GB, the Core absorbs new DMPs. Its mass increases $\propto \tau^{3 / 2}$, and its angular momentum $L_{\text {rot }}$ increases much faster $\propto \tau^{2}$, until it detonates again at the next critical point of its stability;

- Afterglow of GBs is a result of processes developing in the Nuclei and shells after detonation;

- In case of Extrasolar systems, a star wind is the afterglow of star detonation: star Core absorbs new DMPs, increases its mass $\propto \tau^{3 / 2}$ and gets rid of extra $L_{\text {rot }}$ by star wind particles;

- Solar wind is the afterglow of Solar Core detonation 4.6 billion years ago. It creates the bubble of the heliosphere continuously (see Section 6.6);

- In case of Galaxies, a galactic wind is the afterglow of repeating galactic Core detonations. In Milky Way it continuously creates two Dark Matter Fermi Bubbles (see Section 6.7).

S. E. Koposov, et al. present the discovery of the fastest Main Sequence hyper-velocity star S5-HVS1 with mass about 2.3 solar masses that is located at a distance of $\sim 9 \mathrm{kpc}$ from the Sun. When integrated backwards in time, the orbit of the star points unambiguously to the Galactic Centre, implying that S5-HVS1 was kicked away from Sgr $A^{\star}$ with a velocity of $\sim 1800 \mathrm{~km} / \mathrm{s}$ and travelled for 4.8 Myr to the current location. So far, this is the only hyper-velocity star confidently associated with the Galactic Centre [68]. In frames of the developed Model this discovery can be explained by Gravitational Burst of the overspinning Core of the Milky Way 4.8 million years ago, which gave birth to S5-HVS1 with the speed higher than the escape velocity of the Core.

C. J. Clarke, et al. observed CI Tau, a young 2 million years old star. CI Tau is located about 500 light years away in a highly-productive stellar "nursery" region of the galaxy. They discovered that the Extrasolar System contains four gas giant planets that are only 2 million years old [69], amount of time that is too short for formation of gas giants according to prevailing theories.

In frames of the developed Rotational Fission model, this discovery can be explained by Gravitational Burst of the overspinning Core of the Milky Way two million years ago, which gave birth to CI Tau system with all planets generated 
at the same time [11].

To summarize,

- The rotational fission of macroobject Cores is the most probable process that can generate satellite cores with large orbital momenta in a very short time;

- Macrostructures of the World form from the top (superclusters) down to galaxies, extrasolar systems, planets, and moons;

- Gravitational waves can be a product of rotational fission of overspinning Macroobject Cores;

- Hypersphere World-Universe model can serve as a basis for Transient Gravitational Astrophysics.

\subsection{Distribution of the World's Energy Density}

According to WUM, the total Dions relative energy density $\rho_{\text {Dion }}$ in terms of $\rho_{p}$ equals to [11]:

$$
\rho_{\text {Dion }}=\frac{45}{\pi} \rho_{p}=0.68775927 \rho_{c r}
$$

Our Model holds that the energy density of all types of self-annihilating DMPs is proportional to the proton energy density $\rho_{p}$ in the World's Medium. In all, there are 5 different types of self-annihilating DMPs: DMF1, DMF2, DIRAC, ELOP, and DMF3. Then the total energy density of DM $\rho_{D M}$ is

$$
\rho_{D M}=5 \rho_{p}=0.24007327 \rho_{c r}
$$

The total baryonic energy density $\rho_{B}$ is:

$$
\rho_{B}=1.5 \rho_{p}
$$

The sum of electron and MBR energy densities $\rho_{\text {eMBR }}$ equals to:

$$
\rho_{e M B R}=\rho_{e}+\rho_{M B R}=1.5 \frac{m_{e}}{m_{p}} \rho_{p}+2 \frac{m_{e}}{m_{p}} \rho_{p}=3.5 \frac{m_{e}}{m_{p}} \rho_{p}
$$

We take energy density of neutrinos $\rho_{v}$ to equal:

$$
\rho_{v}=\rho_{M B R}
$$

For FIRB radiation energy density $\rho_{\text {FIRB }}$ we take

$$
\rho_{F I R B}=\frac{1}{5 \pi} \frac{m_{e}}{m_{p}} \rho_{p} \approx 0.032 \rho_{M B R}
$$

which corresponds to the value of $0.034 \rho_{M B R}$ calculated by E. L. Wright [70]. Then the energy density of the World $\rho_{W}$ in Luminous Epoch equals to the theoretical critical energy density $\rho_{c r}$

$$
\rho_{W}=\left[\frac{45}{\pi}+6.5+\left(5.5+\frac{1}{5 \pi}\right) \frac{m_{e}}{m_{p}}\right] \rho_{p}=\rho_{c r}
$$

Considering the Equation (3.1.2) for $\rho_{p}$, from this equation we can calculate the value of $1 / \alpha$ using electron-to-proton mass ratio $\frac{m_{e}}{m_{p}}$ 


$$
\frac{1}{\alpha}=\frac{\pi}{15}\left[450+65 \pi+(55 \pi+2) \frac{m_{e}}{m_{p}}\right]=137.03600
$$

which is in excellent agreement with the commonly adopted value of 137.035999. It follows that there is a direct correlation between constants $\alpha$ and $m_{e} / m_{p}$ expressed by the obtained equation. As shown, $m_{e} / m_{p}$ is not an independent constant but is instead derived from $\alpha$ [11].

As the conclusion, according to WUM:

- The World's energy density is inversely proportional to Fundamental parameter $Q$ in all cosmological times;

- The particles relative energy densities are proportional to Fundamental constant $\alpha$ in Luminous Epoch.

\section{Dark Matter Astrophysics}

\subsection{Macroobject Shell Model}

In our view, Macroobjects of the World possess the following properties [8]:

- Nuclei are made up of DMPs. Surrounding shells contain DM and baryonic matter;

- Nuclei and shells are growing in time proportionally to square root of cosmological time $\propto \tau^{1 / 2}$ until one of them reaches the critical point of its local stability, at which it detonates. The energy released during detonation is produced by the annihilation of DMPs. The detonation process does not destroy the Macroobject; instead, Hyper-flares occur in active regions of the shells, analogous to Solar flares;

- All other DMPs in different shells can start annihilation process as the result of the first detonation;

- Different emission lines in spectra of bursts are connected to the Macroobjects' structure which depends on the composition of the Nuclei and surrounding shells made up of DMPs. Consequently, the diversity of Very High Energy Bursts has a clear explanation;

- Afterglow is a result of processes developing in Nuclei and shells after detonation.

\subsection{Multiwavelength Pulsars}

According to WUM, Macroobjects Cores made up of self-annihilating DMF1 and DMF2 have maximum mass and minimum size which are equal to parameters of neutron stars [8]. It follows that Gamma-Ray Pulsars might be, in fact, rotating DMF1 or DMF2 star. The nuclei of such pulsars may also be made up of their mixture surrounded by shells composed of other DMPs. Gamma-Ray Pulsar multiwavelength radiation depends on the composition of Nucleus and shells [8].

S. Ansoldi, et al. report the most energetic pulsed emission ever detected from the Crab pulsar reaching up to $1.5 \mathrm{TeV}$. Such $\mathrm{TeV}$ pulsed quants require a parent population of electrons with a Lorentz factor of at least $5 \times 10^{6}$. These results 
strongly suggest Inverse Compton scattering of low energy photons as the emission mechanism [71].

Ge Chen, et al. report hard X-ray observations of the young rotation-powered radio pulsar PSR B1509 in the range spanning from $3 \mathrm{keV}$ through $500 \mathrm{MeV}$. Astronomers hypothesize that the pulsar's lack of $\mathrm{GeV}$ emission is due to viewing geometry, with the $\mathrm{X}$-rays originating from synchrotron emission from secondary pairs in the magnetosphere [72].

WUM: Very High Energy pulsed emission from the Crab pulsar can be explained by active area of rotating Star composed of a mixture of annihilating DMF1 $(1.3 \mathrm{TeV})$ and DMF2 $(9.6 \mathrm{GeV})$. Multiwavelength emission from pulsar PSR B1509 can be explained by rotating DMF2 star with an active area irradiating gamma quants with energy $9.6 \mathrm{GeV}$, which interact with surrounding shells, causing them to glow in X-ray spectrum [8].

\subsection{Binary Millisecond Pulsars}

The properties of the growing class of radio pulsars with low-mass companions are discussed in literature. S. Johnston, et al. have discovered pulsar PSR J0437-4715 with by far the greatest flux density of any known millisecond pulsar [73]. M. Bailes, et al. report the discovery of millisecond pulsar PSR J2145-0750 that has a spin-down age of approximately greater than $12 \mathrm{Gyr}$ [74]. Binary millisecond pulsar PSR J1311-3430 was explained by a model where mass from a low mass companion is transferred onto the pulsar, increasing the mass of the pulsar and decreasing its period. The averaged gamma-ray spectral energy distribution for the pulsar has cut-off about $10 \mathrm{GeV}$ [75].

WUM: These experimental results can be explained by rotating DMF2 star made up of annihilating DMF2 $(9.6 \mathrm{GeV})$ with mass that is growing in time proportionally to $\propto \tau^{3 / 2}$. DMF2 star is receiving mass and energy at the rate $W_{r} \propto \tau^{1 / 2}$. When the received power $W_{r}$ is greater than the gamma-ray power irradiated by the active area of the rotating DMF2 star, the decreasing of its period will be observed. Then there is no need to introduce a low-mass companion [8].

\subsection{Gamma-Ray Bursts}

Gamma-Ray Bursts (GRBs) status after 50 years of investigations looks as follows [6]:

- The intense radiation of most observed GRBs is believed to be released when a rapidly rotating, high-mass star collapses to form a neutron star, quark star, or black hole;

- Short GRBs appear to originate from merger of binary neutron stars;

- There are seven known soft gamma repeaters. It means that some GRBs are not catastrophic events.

WUM: The experimental results for GRBs have the following explanation [6]:

- Nuclei and shells of galaxies made up of DMPs are responsible for GRBs; 
- GRBs convert energy into radiation through annihilation of DMPs;

- Spectrum of GRBs depends on composition of Nuclei and shells;

- Afterglow is a result of processes developing in the Nuclei and shells after detonation.

\subsection{Young Stellar Object Dippers}

The Mysterious Star KIC $\mathbf{8 4 6 2 8 5 2}$ with its large irregular dimmings is a main-sequence star with a rotation period $\sim 0.88$ day that exhibits no significant Infrared excess. A stellar mass is $M=1.43 M_{\odot}$, luminosity $L=4.68 L_{\odot}$, and radius $R=1.58 R_{\odot}$. While KIC 8462852's age was initially estimated to be hundreds of millions of years, a number of astronomers have argued that it could be much younger-just like EPIC 204278916. Young stars with protoplanetary disks should emit light in the infrared, but observations show no evidence for warm dust, which would exist if a planetary collision debris were at play.

Results obtained by T. S. Boyajian, et al. [76] show that the 0.88-day signal is present in most of the Kepler time series, with the strongest presence occurring around day 1200. Interestingly however, around day 400 and day 1400, T. S. Boyajian, et al. observed major contributions at different frequencies, corresponding to 0.96 days and 0.90 days, respectively. A prominent hypothesis, based on a lack of observed infrared light, posits a swarm of cold, dusty comet fragments in a highly eccentric orbit. However, the notion that disturbed comets from such a cloud could exist in high enough numbers to obscure $22 \%$ of the star's observed luminosity has been doubted [76].

EPIC 204278916 had irregular dimmings of up to $65 \%$ for 25 consecutive days out of 78.8 days of observations. For the remaining duration of the observations, the variability is highly periodic and attributed to stellar rotation. The star is about five million years old with radius $R=0.97 R_{\odot}$ and mass $M \sim 0.5 M_{\odot}$ [77]. S. Scaringi, et al. hypothesize that the irregular dimmings are caused by either a warped inner-disk edge or transiting cometary-like objects in either circular or eccentric orbits. Most of the proposed mechanisms assume nearly edge-on viewing geometries. However, an analysis of the known dippers by M. Ansdell, et al. shows that nearly edge-on viewing geometries are not a defining characteristic of the dippers and that additional models should be explored [78] [79].

EPIC 204376071 is a young $M$ star of mass $0.16 M_{\odot}$ and radius $0.63 R_{\odot}$ that exhibits only a single 80 percent deep occultation of 1 -d duration. The star has frequent flares and a low-amplitude rotational modulation but is otherwise quiet over 160 days of cumulative observation. S. Rappaport, et al. give two possible explanations: orbiting dust or small particles (e.g. a disc bound to a smaller orbiting body, or unbound dust that emanates from such a body) or a transient accretion event of dusty material near the corotation radius of the star [80].

WUM: The experimental results above can be explained as follows [8]:

- The average density of the Dippers is 1.5 - 3 times smaller than the average density of the Sun; 
- Consequently, the density of these stars' Nuclei (made of DMF1 or DMF2) is smaller than nuclear density. This relatively low density makes density fluctuations inside of the Nucleus possible;

- An annihilation of DMF1 or DMF2 depends on a concentration of DMPs squared;

- As the result of the huge density fluctuation, some bulk of the Nucleus can arise in which the annihilation process ceases. It will cause a drop of the star luminosity in this area;

- The Nucleus is rotating ( $\sim 0.88$ days in case of KIC 8462852$)$, and consequently the regular dimming events are observed;

- Change in the position of the huge density fluctuation inside of the Nucleus is responsible for the change of the regular dimming event frequency from $\sim 0.88$ days (around day 1200) to $\sim 0.96$ and $\sim 0.90$ days (around day 400 and day 1400) respectively [76];

- Irregular dimming events are the result of random density fluctuations in the bulk of Nucleus.

\subsection{Multiworld}

In Section 4.3 we introduced Weak interaction with the parameter $G_{W}=G_{O} \times Q^{-1 / 4}$, which is about 30 orders of magnitude greater than $G$. According to Multiworld proposed in WUM [11], Weak interaction defines a Micro-World and its objects with mass about Planck mass are the building blocks of Macroobjects.

Below we discuss the main characteristics of a Large-World and Small-World in the Multiworld based on the proposed Extremely-Weak and Super-Weak interaction respectively. Large-World is characterized by the parameter $G_{E W}=G_{O} \times Q^{-3 / 4}$, which is about 10 orders of magnitude greater than $G$. The range of the extremely-weak interaction $R_{E W}$ in the present epoch equals to [12]:

$$
R_{E W}=a \times Q^{3 / 4}=1.44115 \times 10^{16} \mathrm{~m}=1.5233 \mathrm{ly}
$$

According to WUM, Extrasolar Systems (ESs) are Large-Worlds with a boundary between them and Intergalactic Medium that has a surface energy density $\sigma_{0}$. This vast, bubble-like region of space, which surrounds Sun, is named Heliosphere. The bubble of the heliosphere is continuously inflated by solar jets, known as the solar wind [81]. The outside radius of the solar heliosphere $R_{H S}$ equals to:

$$
R_{H S}=\left(\frac{3 M_{\odot} c^{2}}{4 \pi \sigma_{0}}\right)^{1 / 2} \cong 1.1 \times 10^{15} \mathrm{~m} \cong 0.12 \mathrm{ly}
$$

where $M_{\odot}$ is the mass of the Sun. The value of 3 above follows from the ratio for all Macroobjects of the World: $1 / 3$ of the total mass is in the central macroobject and $2 / 3$ of the total mass is in the structure around it (see Section 7.5). In WUM, ESs have Cores made up of DMPs surrounded by shells composed of 
DM and baryonic matter. Extremely-weak interaction between DM Cores and all particles around them provide integrity of ESs.

Let's calculate parameters of Large Objects (LOs) made up of self-annihilating DMF1 and DMF2 particles, considering extremely-weak interaction between them. WUM develops the mathematical framework that allows for the calculation of these parameters [7]. According to WUM, the maximum mass of Macroobjects (MOs) $M_{M O}^{\max }$ composed of self-annihilating particles DMF1 and DMF2 in the Macro-World can be found by the following equation [7]:

$$
M_{M O}^{\max }=\frac{\pi}{6} \frac{M_{P}^{3}}{m_{p}^{2}}
$$

where Planck mass $M_{P}$ squared equals to:

$$
M_{P}^{2}=\frac{h c}{2 \pi G}=2 m_{0}^{2} \times Q
$$

and $m_{p}$ is proton mass. The minimum radius of these MOs $R_{M O}^{\min }$ equals to:

$$
R_{M O}^{\min }=\frac{M_{P} m_{0}}{2 m_{p}^{2}} a
$$

The maximum density of these MOs $\rho_{M O}^{\max }$ equals to the nuclear density $\rho_{\max }:$

$$
\rho_{\max }=\left(\frac{m_{p}}{m_{0}}\right)^{4} \rho_{0}
$$

In the Large-World, the maximum mass of Large Objects $M_{L O}^{\max }$ made up of self-annihilating particles DMF1 and DMF2 equals to:

$$
M_{L O}^{\max }=\frac{\pi}{6} \frac{M_{E W}^{3}}{m_{p}^{2}}
$$

where mass $M_{E W}$ squared equals to:

$$
M_{E W}^{2}=\frac{h c}{2 \pi G_{E W}}=2 m_{0}^{2} \times Q^{3 / 4}
$$

The minimum radius of these LOs $R_{L O}^{\min }$ equals to:

$$
R_{L O}^{\min }=\frac{M_{E W} m_{0}}{2 m_{p}^{2}} a
$$

with the maximum density $\rho_{L O}^{\max }$ equals to the nuclear density (6.6.1).

Considering the constancy of the product of a mass of $\mathrm{MO}$ and cube of its radius: $M_{M O} \times R_{M O}^{3}=$ const [7] and the minimum density of LO $\rho_{\min }$ that is enough for the self-annihilation of DMPs we can estimate the minimum mass $M_{L O}^{\min }$ and maximum radius $R_{L O}^{\max }$ of LOs:

$$
\begin{aligned}
& M_{L O}^{\min }=\left(\frac{\rho_{\min }}{\rho_{\max }}\right)^{1 / 2} M_{L O}^{\max } \\
& R_{L O}^{\max }=\left(\frac{\rho_{\max }}{\rho_{\min }}\right)^{1 / 6} R_{L O}^{\min }
\end{aligned}
$$


In our opinion, Fermi Bubbles contain Large Objects built up from self-annihilating particles DMF1 and DMF2 (see Section 6.7).

Small-World is characterized by the parameter $G_{S W}=G_{O} \times Q^{-1 / 2}$, which is about 20 orders of magnitude greater than $G$. The range of the super-weak interaction $R_{S W}$ in the present epoch equals

$$
R_{S W}=a \times Q^{1 / 2}=1.54351 \times 10^{6} \mathrm{~m}
$$

In WUM, Ball Lightnings are the objects of Small-Worlds that have cores made up of DMPs surrounded by shells composed of electron-positron plasma. Super-weak interaction between DM cores and all particles around them provide integrity of Ball Lightnings (see Section 7.8).

Parameters of Small Objects made up of self-annihilating particles DMF1 and DMF2 considering super-weak interaction between them can be calculated by the same Equations (6.6.1)-(6.6.6) with the replacement of the mass $M_{E W}$ for the mass $M_{S W}$ that equals to:

$$
M_{S W}^{2}=\frac{h c}{2 \pi G_{S W}}=2 m_{0}^{2} \times Q^{1 / 2}
$$

In our view, Fermi Bubbles also contain Small Objects made up of self-annihilating particles DMF1 and DMF2 (see Section 6.7).

\subsection{Dark Matter Fermi Bubbles}

In November 2010, the discovery of two Fermi Bubbles (FBs) emitting gammaand X-rays was announced. FBs extend for about 25 thousand light years above and below the center of the galaxy [82]. The outlines of the bubbles are quite sharp, and the bubbles themselves glow in nearly uniform gamma rays over their colossal surfaces. Gamma-ray spectrum measured by the Fermi Large Area Telescope at Galactic latitude $\geq 10^{\circ}$ has an exponential cutoff at energies $\sim 100 \mathrm{GeV}$. However, the FBs gamma-ray spectrum at latitude $\leq 10^{\circ}$, without showing any sign of cutoff up to around $1 \mathrm{TeV}$ in the latest tentative results, remains unconstrained [83]. Years after the discovery of FBs, their origin and the nature of the gamma-ray emission remain unresolved.

M. Su and D. P. Finkbeiner identify a gamma-ray cocoon feature in the southern Fermi bubble, a jet-like feature along the cocoon's axis of symmetry, and another directly opposite the Galactic center in the north. Both the cocoon and jet-like feature have a hard spectrum from 1 to $100 \mathrm{GeV}$. If confirmed, these jets are the first resolved gamma-ray jets ever seen [84].

G. Ponti, et al. report prominent X-ray structures on intermediate scales (hundreds of parsecs) above and below the plane, which appear to connect the Galactic Centre region to the Fermi bubbles. They propose that these structures, which they term the Galactic Centre "chimneys", constitute exhaust channels through which energy and mass, injected by a quasi-continuous train of episodic events at the Galactic Centre, are transported from the central few parsecs to the base of the FBs [85].

D. Hooper and T. R. Slatyer discuss two emission mechanisms in the FBs: in- 
verse Compton scattering and annihilating DM [86]. In their opinion, the second emission mechanism must be responsible for the bulk of the low-energy, low-latitude emission. The spectrum and angular distribution of the signal is consistent with that predicted from $\sim 10 \mathrm{GeV}$ DMPs annihilating to leptons. This component is similar to the excess $\mathrm{GeV}$ emission previously reported by $\mathrm{D}$. Hooper from the Galactic Center [87].

It is worth to note that a similar excess of gamma-rays was observed in the central region of the Andromeda galaxy (M31). A. McDaniel, T. Jeltema, and S. Profumo calculated the expected emission across the electromagnetic spectrum in comparison with available observational data from M31 and found that the best fitting models are with the DMP mass $11 \mathrm{GeV}$ [88].

According to H.-Y. Karen Yang, M. Ruszkowski, and E. G. Zweibel, for understanding the physical origin of the FBs, three major questions need to be answered:

- First, what is the emission mechanism? The bubbles can either be hadronic, where the gamma rays are produced by inelastic collisions between cosmic-ray protons and the thermal nuclei via decay of neutral pions, or leptonic, where the gamma rays are generated by inverse-Compton scattering of the interstellar radiation field by cosmic-ray electrons;

- Second, what activity at the Galactic Center triggered the event-are the bubble associated with nuclear star formation or active galactic nucleus activity?

- Third, where are the Cosmic rays accelerated? They could either be accelerated at the Galactic Center and transported to the surface of the bubbles or accelerated in-situ by shocks or turbulence. Note however that not all combinations of the above three considerations would make a successful model because of constraints given by the hard spectrum of the observed bubbles [89].

WUM explains FBs the following way:

- Core of Milky Way galaxy is made up of DM particles: DMF1 (1.3 TeV), DMF2 (9.6 GeV), and DMF3 (3.7 keV). The second component (DMF2) explains the excess $\mathrm{GeV}$ emission reported by Dan Hooper from the Galactic Center [86]. Core rotates with surface speed at equator close to the escape velocity between Gravitational Bursts (GBs), and over the escape velocity at the moments of GBs;

- Bipolar astrophysical jets (which are astronomical phenomena where outflows of matter are emitted as an extended beams along the axis of rotation [90]) of DMPs are ejected from the rotating Core into the Galactic halo along the rotation axis of the Galaxy;

- Due to self-annihilation of DMF1 and DMF2, these beams are gamma-ray jets [84]. The prominent X-ray structures on intermediate scales (hundreds of parsecs) above and below the plane (named the Galactic Centre "chimneys" [85]) are the result of the self-annihilation of DMF3;

- FBs are bubbles with boundary between them and Intergalactic Medium that 
has a surface energy density $\sigma_{0}$. These bubbles are filled with DM particles: DMF1, DMF2, and DMF3. In our Model, FBs are Macroobjects with a mass $M_{F B}$ and diameter $D_{F B}$, which are proportional to: $M_{F B} \propto Q^{3 / 2}$ and $D_{F B} \propto Q^{3 / 4}$ respectively. According to WUM, diameter of FBs equals to:

$$
D_{F B}=L_{D M F 3} \times Q^{3 / 4}=\frac{a}{\alpha^{2}} \times Q^{3 / 4}=28.6 \mathrm{kly}
$$

where $L_{D M F 3}$ is Compton length of particles DMF3 with mass $m_{D M F 3}=\alpha^{2} m_{0}$. The calculated diameter is in good agreement with the measured size of the FBs $25 \mathrm{kly}$ [82] and $32.6 \mathrm{kly}$ [84]. With Nikola Tesla's principle at heart-There is no energy in matter other than that received from the environment-we calculate mass $M_{F B}$ and average density $\rho_{F B}$ :

$$
\begin{gathered}
M_{F B}=\frac{\pi D_{F B}^{2} \sigma_{0}}{c^{2}}=\frac{\pi m_{0}}{\alpha^{4}} \times Q^{3 / 2} \cong 3.6 \times 10^{41} \mathrm{~kg} \\
\rho_{F B}=\frac{6 \sigma_{0}}{D_{F B} c^{2}}=6 \alpha^{2} \rho_{0} \times Q^{-3 / 4}
\end{gathered}
$$

Recall that the mass of Milky Way galaxy $M_{M W}$ is about: $M_{M W} \cong 3.2 \times 10^{42} \mathrm{~kg}$;

- DMF3 $(3.7 \mathrm{keV})$ particles have the smallest mass and hence the largest particle concentration. When the distance between them is less than $R_{W}$ (4.3), weak interaction will provide the integrity of FBs. Let's compare average density of FBs (6.7.1) with the minimum density of the DMF3 $\rho_{D M F 3}$ providing weak interaction:

$$
\rho_{D M F 3}=\frac{m_{D M F 3}}{R_{W}^{3}}=\frac{\alpha^{2} m_{0}}{a^{3} Q^{3 / 4}}=\alpha^{2} \rho_{0} \times Q^{-3 / 4}
$$

Comparison of (6.7.1) with (6.7.2) shows that if the density of the DMF3 particles in FBs is larger than $\frac{1}{6} \rho_{F B}$, then the distance between them is less than $R_{W}$. It is a reasonable assumption considering that the shell of DMF3 particles in the Core of galaxy is the biggest in size and the largest in mass. As the result:

- Weak interaction between DMF3 particles provides integrity of Fermi Bubbles;

- FBs made up of DMF3 particles resembles a honeycomb filled with DMF1 and DMF2;

- FBs radiate $\mathrm{X}$-rays due to the annihilation of DMF3 particles with concentration $n_{D M F 3} \geq R_{W}^{-3}$. Concentrations of DMF1 and DMF2 in FBs are very small: about $\alpha^{3}$ and $\alpha^{4}$ smaller than $n_{D M F 3}$, respectively. In our view, gamma rays up to $1 \mathrm{TeV}$ [83] are the result of annihilation of DMF1 $(1.3 \mathrm{TeV})$ and DMF2 $(9.6 \mathrm{GeV})$ in Dark Matter Objects (DMOs). DMOs are macroobjects whose density is sufficient for the annihilation of DMPs to occur. On the other hand, DMOs are much smaller than stars in the World, and have a high concentration in FBs to provide nearly uniform gamma ray glow over their colossal surfaces; 
- Considering the value of the nuclear density $\rho_{\max }=7.2 \times 10^{17} \mathrm{~kg} / \mathrm{m}^{3} \quad$ [7] and the minimum density of DMOs $\rho_{\min } \cong 10^{3} \mathrm{~kg} / \mathrm{m}^{3} \quad$ [11] we can calculate the parameters of Large Objects (LOs) according to Equations (6.6.1)-(6.6.6):

$$
\begin{aligned}
M_{L O}^{\max } \cong 2.1 \times 10^{15} \mathrm{~kg} \\
M_{L O}^{\min } \cong 7.8 \times 10^{7} \mathrm{~kg} \\
R_{L O}^{\min } \cong 8.9 \times 10^{-2} \mathrm{~m} \\
R_{L O}^{\max } \cong 27 \mathrm{~m}
\end{aligned}
$$

- Following Equations (6.6.1)-(6.6.7), we can calculate parameters of Small Objects (SOs):

$$
\begin{gathered}
M_{S O}^{\max } \cong 2.3 \mathrm{~kg} \\
M_{S O}^{\min } \cong 8.5 \times 10^{-8} \mathrm{~kg} \\
R_{S O}^{\min } \cong 9.2 \times 10^{-7} \mathrm{~m} \\
R_{S O}^{\max } \cong 2.7 \times 10^{-4} \mathrm{~m}
\end{gathered}
$$

It is worth to note that in WUM Small Objects are macroobjects with mass larger than Planck mass [5].

- The total flux of the gamma radiation from FBs is the sum of the contributions of all individual LOs and SOs. Their abundance: $\left(10^{26}-10^{33}\right)$ LOs and $\left(10^{34}-10^{41}\right)$ SOs and uniform distribution explain the nearly uniform gamma ray glow of Fermi Bubbles over their colossal surfaces [84]. The LOs and SOs irradiate gamma quants with different energies and attract new DMF1 and DMF2 from BFs due to super-weak interaction. The Core of the Milky Way supplies FBs with new DMPs through the galactic wind, explaining the brightness of FBs remaining fairly constant during the time of observations. In our opinion, FBs are built continuously throughout the lifetime of Milky Way (13.8 By).

In our view, FBs are DMP clouds containing uniformly distributed clumps of Small Objects and Large Objects, in which DMPs annihilate and radiate X-rays and gamma rays. Dark Matter Fermi Bubbles constitute a principal proof of the World-Universe Model.

\section{Solar System}

The most widely accepted model of Solar System formation, known as the Nebular hypothesis, was first proposed in 1734 by Emanuel Swedenborg [91], and later elaborated and expanded upon by Immanuel Kant in 1755 in his "Universal Natural History and Theory of the Heavens" [92].

Lunar origin fission hypothesis was proposed by George Darwin in 1879 to explain the origin of the Moon by rapidly spinning Earth, on which equatorial gravitative attraction was nearly overcome by centrifugal force [93]. Donald U. Wise made a detailed analysis of this hypothesis in 1966 and concluded that "it 
might seem prudent to include some modified form of rotational fission among our working hypothesis" [94].

Solar fission theory was proposed by Louis Jacot in 1951 [95]. Tom Van Flandern further extended this theory in 1993 [96]. Neither L. Jacot nor T. Van Flandern proposed an origin for the Sun itself. It seems that they followed the standard Nebular hypothesis of formation of the Sun. In WUM we concentrate on furthering the Solar Fission theory [11].

Not one of existing models solves the Angular Momentum problem-why is the orbital momentum of Jupiter larger than rotational momentum of the Sun?

\subsection{Angular Momentum}

Considering rotational and orbital angular momentum of all gravitationally-rounded objects in the Solar system, from Mimas, a small moon of Saturn $\left(3.75 \times 10^{19} \mathrm{~kg}\right)$, to the Sun itself $\left(2 \times 10^{30} \mathrm{~kg}\right)$ [11], we find that

- The rotational momentum of the Sun is smaller than Jupiter's, Saturn's, Uranus's, and Neptune's orbital momentum;

- The rotational momentum of the Earth is substantially smaller than Moon's orbital momentum.

From the point of view of Fission model, the prime object is transferring some of its rotational momentum to orbital momentum of the satellite. It follows that at the moment of creation the rotational momentum of the prime object should exceed the orbital momentum of its satellite.

As we pointed out in Section 5.2, Extrasolar system Cores made up of DMFs can give birth to planetary cores, and they can generate cores of moons through the same Rotational Fission mechanism. Let's analyze this possibility for the Solar System.

The Solar system was born 4.6 billion years ago as the result of a Gravitational burst of Milky Way's Core. At that time, Age parameter $\theta_{9.6}$ equaled about $\cong 2 / 3$, and the rotational angular momentum of the Core $L_{r o t}^{M W C}$ was (see Equation (5.2)):

$$
L_{r o t}^{M W C}=1.4 \times 10^{63} \mathrm{~J} \cdot \mathrm{s}
$$

At that time, the Galactic Core could generate approximately $\sim 10^{7}$ Extrasolar systems like the Solar system. Considering that Jupiter's orbital momentum is about $60 \%$ of the total angular momentum of Solar System $L_{t o t}^{S S}$, we obtain for $L_{\text {tot }}^{S S}$ :

$$
L_{\text {tot }}^{S S} \cong 3.2 \times 10^{43} \mathrm{~J} \cdot \mathrm{S}
$$

Let's calculate parameters of the Sun's Core necessary to provide this angular momentum. Substituting mass $M_{\odot}=2 \times 10^{30} \mathrm{~kg}$ and radius $R_{\odot}=7 \times 10^{8} \mathrm{~m}$ and using Equation (5.2) we obtain

$$
L_{\text {rot }}^{\text {Sun }}=1.1 \times 10^{44} \mathrm{~J} \cdot \mathrm{S}
$$

which is 3.3 times greater than $L_{t o t}^{S S}$. It follows that the Sun's Core can be smaller. 
Let's consider the structure of the Sun. According to the standard Solar model it has:

- Nucleus that extends from the center to about $20 \%-25 \%$ of the solar radius, contains $34 \%$ of the Sun's mass with density $\rho_{\max }=1.5 \times 10^{5} \mathrm{~kg} / \mathrm{m}^{3}$ and $\rho_{\min }=2 \times 10^{4} \mathrm{~kg} / \mathrm{m}^{3}$. It produces all Sun's energy;

- Radiative zone from the center to about $70 \%$ of the solar radius with density $\rho_{\text {max }}=2 \times 10^{4} \mathrm{~kg} / \mathrm{m}^{3}$ and $\rho_{\min }=2 \times 10^{2} \mathrm{~kg} / \mathrm{m}^{3}$ in which convection does not occur and energy transfer occurs by means of radiation;

- Nucleus and Radiative zone contain practically all Sun's mass [97].

In our opinion, the Sun has an Inner Core (Nucleus made up of DMF1) whose radius is $20 \%-25 \%$ of the solar radius, and an Outer Core-the Radiative zone. We then calculate the Solar Core rotational angular momentum $L_{\text {rot }}^{S C}$ :

$$
L_{r o t}^{S C} \cong 8.9 \times 10^{43} \mathrm{~J} \cdot \mathrm{s}
$$

which is 2.8 times larger than the overall angular momentum of the Solar System.

Let's follow the same procedure for the Earth-Moon pair. Considering the mass of Earth $M_{\oplus}=6 \times 10^{24} \mathrm{~kg}$, radius $R_{\oplus}=6.4 \times 10^{6} \mathrm{~m}, \quad \theta_{9.6} \cong 2 / 3, \delta=2.9 / 13.1$, we calculate

$$
L_{\text {rot }}^{\text {Earth }}=6.6 \times 10^{34} \mathrm{~J} \cdot \mathrm{s}
$$

that is 2.3 times larger than the Moon's orbital momentum $L_{\text {orb }}^{\text {Moon }}=2.9 \times 10^{34} \mathrm{~J} \cdot \mathrm{s}$ [11].

Let's contemplate the structure of the Earth. According to the standard model, it is composed of:

- An inner core and an outer core that extend from the center to about $45 \%$ of the Earth radius with density $\rho_{\max }=1.3 \times 10^{4} \mathrm{~kg} / \mathrm{m}^{3}$ and $\rho_{\min }=9.9 \times 10^{3} \mathrm{~kg} / \mathrm{m}^{3}$;

- Lower mantle, spanning from the outer core to about $90 \%$ of the Earth radius (below $660 \mathrm{~km}$ ) with density $\rho_{\max }=5.6 \times 10^{3} \mathrm{~kg} / \mathrm{m}^{3}$ and $\rho_{\min }=4.4 \times 10^{3} \mathrm{~kg} / \mathrm{m}^{3}$;

- Inner core, outer core, and lower mantle contain practically all of the Earth's mass [98].

Very little is known about the lower mantle apart from that it appears to be relatively seismically homogeneous. Outer core-lower mantle boundary has a sharp drop of density $(9.9 \rightarrow 5.6) \times 10^{3} \mathrm{~kg} / \mathrm{m}^{3} \quad$ [98].

In our opinion, lower mantle is a part of the Earth's core. It could be significantly different 4.6 billion years ago, since during this time it was gradually filled with all chemical elements produced by Earth's core due to DMF1 annihilation. Considering the Earth's core with radius $R_{\text {core }}^{\text {Earth }}=5.7 \times 10^{6} \mathrm{~m}\left(\theta_{9.6} \cong 2 / 3\right.$ and $\delta=4.4 / 13.1$ ), the rotational angular momentum equals to:

$$
L_{\text {rot }}^{E C}=6.5 \times 10^{34} \mathrm{~J} \cdot \mathrm{s}
$$

which is 2.2 times larger than the orbital momentum of the Moon.

As the conclusion, the overspinning Core of the Sun can give birth to planetary cores, and they can generate cores of moons through the Rotational Fission mechanism [11]. 


\subsection{Dark Matter Cores of Macroobjects}

The following facts support the existence of DM Cores in Macroobjects [11]:

- Fossat, et al. found that Solar Core rotates $3.8 \pm 0.1$ faster than the surrounding envelope [99];

- By analyzing the earthquake doublets, Zhang, et al. concluded that the Earth's inner core is rotating faster than its surface by about $0.3-0.5$ degrees per year [100].

The fact that Macroobject Cores rotate faster than surrounding envelopes, despite high viscosity of the internal medium, is intriguing. WUM explains this phenomenon through absorption of DMPs by Cores. Dark Matter particles supply not only additional mass $\left(\propto \tau^{3 / 2}\right)$, but also additional angular momentum $\left(\propto \tau^{2}\right)$. Cores irradiate products of annihilation, which carry away excessive angular momentum. The Solar wind is the result of this mechanism.

\subsection{Gravitationally-Rounded Objects Internal Heat}

Earth. The analysis of Sun's heat for planets in Solar system yields the effective temperature of Earth of $255 \mathrm{~K}$ [101]. The actual mean surface temperature of Earth is $288 \mathrm{~K}$ [102]. The higher actual temperature of Earth is due to energy generated internally by the planet itself. According to the standard model, the Earth's internal heat is produced mostly through radioactive decay. The major heat-producing isotopes within Earth are K-40, U-238, and Th-232. The mean global heat loss from Earth is 44.2 TW [103]. The Earth's Uranium has been thought to be produced in one or more supernovae over 6 billion years ago [104].

Radiogenic decay can be estimated from the flux of geoneutrinos that are emitted during radioactive decay. The KamLAND Collaboration combined precise measurements of the geoneutrino flux from the Kamioka Liquid-Scintillator Antineutrino Detector, Japan, with existing measurements from the Borexino detector, Italy. They found that decay of U-238 and Th-232 together contributes about $20 \mathrm{TW}$ to the total heat flux from the Earth to space. The neutrinos emitted from the decay of K-40 contribute $4 \mathrm{TW}$. Based on the observations the KamLAND Collaboration made a conclusion that heat from radioactive decay contributes about half of Earth's total heat flux [105].

Plutonium-244 with half-life of 80 million years is not produced in significant quantities by the nuclear fuel cycle, because it needs very high neutron flux environments. Any Plutonium-244 present in the Earth's crust should have decayed by now. Nevertheless, D. C. Hoffman, et al. in 1971 obtained the first indication of Pu-244 present existence in Nature [106].

In our opinion, all chemical products of the Earth including isotopes K-40, $\mathrm{U}-238$, Th-232, and $\mathrm{Pu}-244$, are produced within the Earth as the result of DMF1 annihilation [11]. They arrive in the Crust of the Earth due to convection currents in the mantle carrying heat and isotopes from the interior to the planet's surface [107]. 
Jupiter radiates more heat than it receives from the Sun [108]. Giant planets like Jupiter are hundreds of degrees warmer than current temperature models predict. Until now, the extremely warm temperatures observed in Jupiter's atmosphere (about 970 degrees C [109]) have been difficult to explain, due to lack of a known heat source [12]. Saturn radiates 2.5 times more energy than it receives from the Sun [110]; Uranus-1.1 times [111]; Neptune-2.6 times [112].

S. Kamata, et al. report that "many icy Solar System bodies possess subsurface oceans. To maintain an ocean, Pluto needs to retain heat inside". Kamata, et al. show that "the presence of a thin layer of gas hydrates at the base of the ice shell can explain both the long-term survival of the ocean and the maintenance of shell thickness contrasts. Gas hydrates act as a thermal insulator, preventing the ocean from completely freezing while keeping the ice shell cold and immobile. The most likely guest gas is methane" [113].

According to WUM, the internal heating of all gravitationally-rounded objects of the Solar system is due to DMPs annihilation in their cores made up of DMF1 $(1.3 \mathrm{TeV})$. The amount of energy produced due to this process is sufficiently high to heat up the objects. New DMF1 freely penetrate through the entire objects' envelope, get absorbed into the cores, and continuously support DMF1 annihilation. Objects' cores are essentially Dark Matter Reactors fueled by DMF1 [11].

In our opinion, all chemical elements are produced by Macroobjects themselves as the result of DMPs annihilation. The diversity of all gravitationally-rounded objects of the Solar System is explained by the differences in their cores (mass, size, composition). The DM Reactors inside of all gravitationally-rounded objects (including Earth) provide sufficient energy for all geological processes on planets and moons. All gravitationally-rounded objects in hydrostatic equilibrium, down to Mimas in Solar system, prove the validity of WUM [11].

\subsection{The Evolution of the Sun}

By 1950s, stellar astrophysicists had worked out the physical principles governing the structure and evolution of stars [114]. According to these principles, the Sun's luminosity had to change over time, with the young Sun being about $30 \%$ less luminous than today. The long-term evolution of the bolometric solar luminosity $L(\tau)$ as a function of cosmological time $\tau$ can be approximated by simple linear law: $L(\tau) \propto \tau$ [114] [115] [116] [117] [118].

One of the consequences of WUM holds that all stars were fainter in the past. As their Cores absorb new DM, size of MO Cores $R_{M O}$ and their luminosity $L_{M O}$ are increasing in time: $R_{M O} \propto \tau^{1 / 2}$ and $L_{M O} \propto R_{M O}^{2} \propto \tau$ respectively. Taking the age of the World $A_{W} \cong 14.2 \mathrm{Byr}$ and the age of the solar system $A_{S S} \cong 4.6 \mathrm{Byr}$, it is easy to find that the young Sun's output was $67 \%$ of what it is today [12]. Literature commonly refers to the value of 70\% [117] [118]. This result supports the developed model of the structure and evolution of the Sun [114]. 


\subsection{Pioneer Anomaly}

According to Fractal Cosmology, Macroobjects are surrounded by transitional regions, in which the density decreases rapidly to the point of the zero level of the fractal structure [119] characterized by radius $R_{f}$ and density $\rho_{f}$, that satisfy the following equation for $r \geq R_{f}$ :

$$
\rho(r)=\frac{\rho_{f} R_{f}}{r}
$$

According to Yu. Baryshev: For a structure with fractal dimension $D=2$ the constant $\rho_{f} R_{f}$ may be actually viewed as a new fundamental physical constant [119]. In WUM, it is natural to connect this constant with a basic unit of energy density $\sigma_{0}[11]$ :

$$
\rho_{f} R_{f}=4 \sigma_{0} / c^{2}
$$

Pioneer anomaly is an observed deviation from predicted acceleration of Pioneer 10 and Pioneer 11 spacecrafts, after they passed about 20 astronomical units on their trajectories out of the Solar System. An unexplained force appeared to cause an approximately constant sunward acceleration of $a_{P}=(8.74 \pm 1.33) \times 10^{-10} \mathrm{~m} / \mathrm{s}^{2}$ for both spacecrafts.

Let us calculate deceleration $a_{P}$ at the distance $r_{P} \gg R_{f}$ due to additional mass of the structure $M_{F S} \propto r_{P}^{2}$ :

$$
a_{P}=\frac{G M_{F S}}{r_{P}^{2}}=c H_{0}=6.68 \times 10^{-10} \mathrm{~m} / \mathrm{s}^{2}
$$

which is in good agreement with the experimentally measured value $\left(R_{0}\right.$ and $H_{0}$ are the values of the World's size $R$ and Hubble's parameter $H$ at the present Epoch). It is important to notice that the calculated deceleration does not depend on $r_{P}$ and equals to $\mathrm{cH}_{0}$ for all objects around the Macroobject at the distance $r \gg R_{f}$. Mass of the structure around Sun $M_{V}$ at distance to Voyager 1 $R_{V} \cong 1.8 \times 10^{13} \mathrm{~m} \quad[120]$ is $\sim 0.15 \% M_{\text {Sun }}$ [11].

\subsection{Solar Corona}

According to the standard model, the visible surface of the Sun, the photosphere, is the layer below which the Sun becomes opaque to visible light. Above the photosphere visible sunlight is free to propagate into space, and almost all of its energy escapes the Sun entirely. Above the photosphere lies the chromosphere that is about $2500 \mathrm{~km}$ thick with temperature that increases gradually with altitude to around $2 \times 10^{4} \mathrm{~K}$ near the top [121]. The particle density decreases rapidly from $10^{22}$ to $10^{17} \mathrm{~m}^{-3}$. Above the chromosphere, in a thin (about $200 \mathrm{~km}$ ) transition region, the temperature rises rapidly from around $2 \times 10^{4} \mathrm{~K}$ in the upper chromosphere to coronal temperatures closer to $10^{6} \mathrm{~K}$. The particle density decreases from $10^{17}$ up to $10^{16}-10^{15} \mathrm{~m}^{-3}$ in the low corona. In our opinion, this is a zero level of the fractal structure. The calculated density according to Equation (7.5.2) is: 


$$
\rho_{f} \cong 2.3 \times 10^{-9} \mathrm{~kg} / \mathrm{m}^{3}
$$

Corona is an aura of plasma that surrounds the Sun and other stars. The Sun's corona extends at least 8 million kilometers into outer space [122] and is most easily seen during a total solar eclipse. Spectroscopy measurements indicate strong ionization and plasma temperature in excess of $10^{6} \mathrm{~K}$ [123]. The corona emits radiation mainly in the $\mathrm{X}$-rays, observable only from space. The plasma is transparent to its own radiation and to solar radiation passing through it, therefore we say that it is optically-thin. The gas, in fact, is very rarefied, and the photon mean free-path by far overcomes all other length-scales, including the typical sizes of the coronal features.

J. T. Schmelz made the following comment on the composition of Solar corona: Along with temperature and density, the elemental abundance is a basic parameter required by astronomers to understand and model any physical system. The abundances of the solar corona are known to differ from those of the solar photosphere [124].

Coronal heating problem in solar physics relates to the question of why the temperature of the Solar corona is millions of degrees higher than that of the photosphere. The high temperatures require energy to be carried from the solar interior to the corona by non-thermal processes.

In our opinion, the origin of the Solar corona plasma is not the coronal heating. Plasma particles (electrons, protons, multicharged ions) are so far apart that plasma temperature in the usual sense is not very meaningful. The plasma is the result of annihilation of DMF1 (1.3 TeV), DMF2 (9.6 GeV), and DMF3 (3.7 keV) particles. The Solar corona made up of DMPs resembles a honeycomb filled with plasma [11].

The following experimental results speak in favor of this model [11]:

- The corona emits radiation mainly in the X-rays due to the annihilation of DMF3;

- The plasma is transparent to its own radiation and to the radiation coming from below;

- The elemental composition of the Solar corona and the Solar photosphere are known to differ;

- During the impulsive stage of Solar flares, radio waves, hard X-rays, and gamma rays with energy above $100 \mathrm{GeV}$ are emitted [125]. In our view, it is the result of enormous density fluctuations of DMF1 and DMF2 in the Solar corona and their annihilation;

- Assuming the particle density in the low corona $10^{15} \mathrm{~m}^{-3}$ and mass of DMF1: $m_{D M F 1}=2.3 \times 10^{-24} \mathrm{~kg}$ we can find mass density $\rho_{D M F 1}^{i n}=2.3 \times 10^{-9} \mathrm{~kg} / \mathrm{m}^{3}$ that is equal to the density of the fractal structure (7.6.1);

- A distance between DMF1 is about $10^{-5} \mathrm{~m}$ that is much smaller than the range of the weak interaction of DMPs $R_{W}$ (4.3);

- At the same density of the fractal structure, distance between DMF2 with mass $m_{D M F 2}=1.7 \times 10^{-26} \mathrm{~kg}$ is about $10^{-6} \mathrm{~m}$, and DMF3 with mass 
$m_{D M F 3}=6.7 \times 10^{-33} \mathrm{~kg}$ is about $10^{-8} \mathrm{~m}$. The smallest distance between DMF3 particles explains the fact that corona emits radiation mainly in the X-rays;

- The Solar corona is a stable Shell around the Sun with density decreasing according to Equation (7.5.1) with inner radius $R_{\text {in }} \cong 7 \times 10^{8} \mathrm{~m}$, and outer radius $R_{\text {out }}: R_{\text {out }}=4 \alpha^{2} a \times Q^{3 / 4} \cong 3 \times 10^{12} \mathrm{~m}$;

- The total mass of the Solar Corona $M_{S C}: M_{S C}=\frac{8 \pi \sigma_{0}}{c^{2}} R_{\text {out }}^{2} \cong 9 \times 10^{25} \mathrm{~kg}$.

\subsection{Geocorona and Planetary Coronas}

The geocorona is the luminous part of the outermost region of the Earth's atmosphere that extends to at least $640,000 \mathrm{~km}$ from the Earth [126]. It is seen primarily via far-ultraviolet light (Lyman-alpha) from the Sun that is scattered by neutral hydrogen.

Far-ultraviolet photons in the geocorona have been observed out to a distance of approximately 100,000 km from the Earth [127]. The first high-quality and wide-field-of-view image of Earth's corona of 243,000 km was obtained by Hisaki, the first interplanetary microspacecraft [128]. Hisaki with its extreme ultraviolet spectrometer EXCEED acquires spectral images $(52-148 \mathrm{~nm}$ ) of the atmospheres of planets from Earth orbit and has provided quasi-continuous remote sensing observations of the geocorona since 2013 [129]. The most popular explanation of this geocoronal emission is the scattering of Solar Far-Ultraviolet (FUV) photons by exospheric hydrogen.

$\mathrm{X}$-rays from Earth's geocorona were first detected by Chandra X-ray Observatory in 1999 [130]. X-rays were observed in the range of energies $0.08-10$ $\mathrm{keV}$ [129]. The main mechanism explaining the geocoronal X-rays is that they are caused by collisions between neutral atoms in the geocorona with carbon, oxygen and nitrogen ions that are streaming away from the Sun in the solar wind [130] [131] [132]. This process is called "charge exchange", since an electron is exchanged between neutral atoms in geocorona and ions in the solar wind.

X-rays from planets were also observed by Chandra [130]. According to NASA:

- The X-rays from Venus and, to some extent, the Earth, are due to the fluorescence of solar X-rays striking the atmosphere;

- Fluorescent X-rays from oxygen atoms in the Martian atmosphere probe heights similar to those on Venus. A huge Martian dust storm was in progress when the Chandra observations were made. The intensity of the X-rays did not change during the dust storm;

- Jupiter has an environment capable of producing X-rays in a different manner because of its substantial magnetic field. X-rays are produced when high-energy particles from the Sun get trapped in its magnetic field and accelerated toward the polar regions where they collide with atoms in Jupiter's atmosphere;

- Like Jupiter, Saturn has a strong magnetic field, so it was expected that Sa- 
turn would also show a concentration of X-rays toward the poles. However, Chandra's observation revealed instead an increased X-ray brightness in the equatorial region. Furthermore, Saturn's X-ray spectrum was found to be similar to that of X-rays from the Sun.

V. I. Shematovich and D. V. Bisikalo gave the following explanation of the planetary coronas [133]: The measurements reveal that planetary coronas contain both a fraction of thermal neutral particles with a mean kinetic energy corresponding to the exospheric temperature and a fraction of hot neutral particles with mean kinetic energy much higher than the exospheric temperature. These suprathermal (hot) atoms and molecules are a direct manifestation of the non-thermal processes taking place in the atmospheres.

In our opinion, the Planetary Coronas are similar to the Solar Corona [11]:

- At the distance of $640,000 \mathrm{~km}$ from the Earth [126], atoms and molecules are so far apart that they can travel hundreds of kilometers without colliding with one another. Thus, the exosphere no longer behaves like a gas, and the particles constantly escape into space. In our view, FUV radiation and X-rays are the consequence of DMF3 annihilation;

- All planets and some observed moons (Europa, Io, Io Plasma Torus, Titan) have X-rays in upper atmosphere of the planets, similar to the Solar Corona;

- The calculated density of the Earth's fractal structure $\rho_{f} \cong 2.5 \times 10^{-7} \mathrm{~kg} / \mathrm{m}^{3}$ is in good agreement with experimental results for atmosphere density at 100 $\mathrm{km}$ altitude [12];

- The Geocorona is a stable Shell around the Earth with inner radius $R_{\text {in }} \cong 6.5 \times 10^{6} \mathrm{~m}$ and observed outer radius $R_{\text {out }} \cong 6.4 \times 10^{8} \mathrm{~m}$. The total mass of this Shell $\cong 4.1 \times 10^{18} \mathrm{~kg}$;

- Suprathermal atoms and molecules proposed by V. I. Shematovich and D. V. Bisikalo are the result of DMPs annihilation in Geocorona, similar to that of Solar corona.

\subsection{High-Energy Atmospheric Physics. Ball Lightning}

Lightning Initiation Problem. Years of balloon, aircraft, and rocket observations have never found large enough electric fields inside thunderstorms to make a spark. And yet lightnings strike the Earth about 4 million times per day. This has led to the cosmic-ray model of lightning initiation [134] [135].

Terrestrial Gamma-Ray Flashes (TGFs) were first detected by chance by NASA's Earth-orbiting Compton gamma ray telescope. Compton was searching for GRBs from exploding stars, when it unexpectedly began detecting very strong bursts of high energy X-rays and gamma rays, coming from Earth [130].

There are two leading models of TGF formation: Lightning leader emission and Dark Lightning [134], but they still don't account for

- A bright TGF observed by a spacecraft in the middle of Sahara Desert on a nice day. The nearest thunderstorms were 1000 miles away [136];

- An ultraviolet telescope installed on the Russian satellite Lomonosov has registered several powerful explosions of light in the Earth's atmosphere at an 
altitude of several dozen kilometers in clear weather [137].

Additionally, in frames of existing models it is difficult to explain the following results [12]:

- Unusual surges of radiation at $511 \mathrm{keV}$ when there were no thunderstorms;

- Beams of antimatter (positrons) produced above thunderstorms on Earth;

- A gamma-ray flash coming down from the overhead thundercloud;

- Some lightnings produce X-rays and others do not;

- Explosive production of energetic particles observed from space;

- The spectra of TGFs at very high energies (40-100 MeV).

According to WUM, the characteristics of Geocorona are similar to the characteristics of the Solar Corona (see Section 7.6). As the result of a large fluctuation of DMPs in Geocorona and their annihilation, X-rays and gamma-rays are going not only up and out of the Earth, but also down to the Earth's surface. In our view, TGFs are, in fact, well-known GRBs [7]. The spectra of TGFs at very high energies can be explained by DMF1 and DMF2 annihilation. Lightning initiation problem can be solved by X-rays and gamma-rays, which slam into the thunderclouds and carve a conductive path through a thunderstorm. From this point of view, it is easy to explain all experimental results summarized above.

Short History of Ball Lightning Hypothesis. Ball lightning (BL) is an unexplained atmospheric phenomenon that is usually associated with thunderstorms and lasts considerably longer than the split-second flash of a lightning bolt. BL usually appears during thunderstorms, sometimes within a few seconds of lightning, but sometimes without apparent connection to a lightning bolt. Different hypothesis were proposed to explain BL, but no one explanation is widely accepted at present:

- Vacuum hypothesis by Nikola Tesla [138] [139];

- Microwave cavity hypothesis by Peter Kapitsa [140];

- Maser-Soliton hypothesis by Peter H. Handel [141];

- Antimatter hypothesis by David Ashby and Colin Whitehead [142];

- Black hole hypothesis by Mario Rabinowitz [143];

- Extreme Ball Lightning hypothesis by Van Devender [144] [145];

- Microwave Bubble hypothesis by H.-C. Wu [146].

According to A. G. Oreshko, " $P$. L. Kapitsa supposed that a ball lightning is a window in another world" [147]. In WUM, it was suggested that BL is an object of the Small-World [12].

Observation of the Optical and Spectral Characteristics of BL was conducted by Jianyong Cen, et al. in 2012 [148]. At a distance of $900 \mathrm{~m}$ a total of 1.64 seconds of digital video of the BL and its spectrum was obtained, from the formation of the $\mathrm{BL}$ after the ordinary lightning struck the ground, up to the optical decay of the phenomenon. The BL traveled horizontally across the video frame at an average speed of $8.6 \mathrm{~m} / \mathrm{s}$. It had a diameter of $5 \mathrm{~m}$.

Ball Lightning Formation. The clue of our model comes from the observed ability of BLs to penetrate solid materials. It means that the core of BL should be composed of DMPs. In WUM, they are DMF1 and DMF2. Small Objects made 
up of self-annihilating particles DMF1 or DMF2 can form cores of BLs in Small-Worlds characterized by super-weak interaction (see Section 6.6).

Following Tesla vacuum hypothesis [138] [139], we suppose that when sudden and very powerful TGF passes through the air and strike the surface of the Earth, "the tremendous expansion of some portions of the air and subsequent rapid cooling and condensation gives rise to the creation of partial vacua in the places of greatest development of heat. These vacuous spaces, owing to the properties of the gas, are most likely to assume the shape of hollow spheres when, upon cooling, the air from all around rushes in to fill the cavity created by the explosive dilatation and subsequent contraction".

In our Model, the places of greatest development of heat are the spots on the Earth's surface struck by TGFs. As the result, the ablation of the soil takes place and vaporized chemical elements of soil and air can be absorbed by BLs and observed experimentally [148].

Very powerful gamma quants with energy of at least $1.02 \mathrm{MeV}$ in the vicinity of atomic nuclei of the ground can produce electron-positron pairs with high concentration. This electron-positron plasma composes a shell around DM core of BL made up of DMF1 or DMF2 and provides their affinity for metal objects such as wires [148].

The most important part of the $\mathrm{BL}$ formation is a $\mathrm{DM}$ core. The calculated density of the Geocorona composed of DMF1 $\rho_{D M F 1}$ near the surface of the Earth is [12]:

$$
\rho_{D M F 1} \cong 2.5 \times 10^{-7} \mathrm{~kg} / \mathrm{m}^{3}
$$

When powerful TGF strikes the surface of the Earth, the explosive dilatation of some portion of Geocorona gives rise to the creation of hollow sphere with partial vacua and all DMPs outside of the sphere. The subsequent rapid contraction induces DMPs rush in to fill the cavity. As the result, at the center of the sphere arises microobject with density high enough for the beginning of the DMPs annihilation. The described microobject attracts new DMPs from Geocorona due to super-weak interaction and grows up to the macroobject with a mass about $\sim 10^{-6} \mathrm{~kg}$ that will start attracting electron-positron pairs produced by TGF [12].

According to WUM, mass of BL's core can grow up to $2.3 \mathrm{~kg}$, and the radius of plasma shell can reach a few meters [12]. Mass of a small BL is mostly concentrated in its DM core. A small BL can thus easily penetrate through walls, glass and metal, generally without leaving a hole. Practically all mass of large BLs is in the plasma. The BL with diameter $5 \mathrm{~m}$ observed by J. Cen, et al. [148] had the mass of about $83 \mathrm{~kg}$ [12].

As the conclusion:

- BL has a core made up of DMF1 or DMF2 surrounded by the electron-positron plasma contaminated by chemical elements of soil and air as the result of TGF strike of the ground; 
- Super-weak interaction between DM core and all particles around it provides integrity of BL;

- The core of BL irradiates quants with different energies and attracts new DMPs from Geocorona due to super-weak interaction. It explains the observed result that the brightness of BL remains fairly constant during its lifetime.

It is important to emphasize that the initial energy required for a $\mathrm{BL}$ creation is insufficient for its sustenance of up to 1200 seconds. Additional energy, therefore, must be consumed by a BL once it had been formed. Once we master the creation of BLs in a controlled environment, we can concentrate our efforts on harvesting that energy. World-Universe Model can serve as a basis for High-Energy Atmospheric Physics.

\section{Conclusions}

Dark Matter is abundant:

- $2.4 \%$ of Luminous Matter is in Superclusters, Galaxies, Stars, Planets, etc.

- $4.8 \%$ of Luminous Matter is in the Medium of the World;

- The remaining $92.8 \%$ of mass is Dark Matter.

Dark Matter is omnipresent:

- Cores of all Macroobjects;

- Coronas of all Macroobjects of the World;

- Fermi Bubbles;

- The Medium of the World;

- Dark Matter Reactors in Cores of all gravitationally-rounded Macroobjects.

Based on the totality of the results obtained by WUM, we suggest adopting existence of Dark Matter in the World from the Classical Physics point of view. While WUM needs significant further elaboration, it can already serve as a basis for a New Physics proposed by Paul Dirac in 1937.

\section{Acknowledgements}

I am a Doctor of Sciences in Physics. I belong to the school of physicists established by Alexander Prokhorov-Nobel Prize Laureate in Physics. I am an author of more than 150 published papers, mostly in the area of Laser Physics. I am eternally grateful to Prof. A. M. Prokhorov and Prof. A. A. Manenkov, whose influence on my scientific life has been decisive.

For 17 years I have developed a model I dubbed the World-Universe Model and published a serious of papers in the Journal of High Energy Physics, Gravitation and Cosmology (JHEPGC). I am much obliged to Prof. C. Corda for publishing my manuscripts in JHEPGC.

Many thanks to my long-term friend Felix Lev for stimulating discussions of history and philosophy of Physics and important comments on the Model. Special thanks to my son Ilya Netchitailo who questioned every aspect of the Model, gave valuable suggestions and helped shape it to its present form. 


\section{Conflicts of Interest}

The author declares no conflicts of interest regarding the publication of this paper.

\section{References}

[1] Netchitailo, V. (2015) 5D World-Universe Model Space-Time-Energy. Journal of High Energy Physics, Gravitation and Cosmology, 1, 25-34. https://doi.org/10.4236/jhepgc.2015.11003

[2] Netchitailo, V. (2015) 5D World-Universe Model. Multicomponent Dark Matter. Journal of High Energy Physics, Gravitation and Cosmology, 1, 55-71. https://doi.org/10.4236/jhepgc.2015.12006

[3] Netchitailo, V. (2016) 5D World-Universe Model. Neutrinos. The World. Journal of High Energy Physics, Gravitation and Cosmology, 2, 1-18. https://doi.org/10.4236/jhepgc.2016.21001

[4] Netchitailo, V. (2016) 5D World-Universe Model. Gravitation. Journal of High Energy Physics, Gravitation and Cosmology, 2, 328-343. https://doi.org/10.4236/jhepgc.2016.23031

[5] Netchitailo, V. (2016) Overview of Hypersphere World-Universe Model. Journal of High Energy Physics, Gravitation and Cosmology, 2, 593-632. https://doi.org/10.4236/jhepgc.2016.24052

[6] Netchitailo, V. (2017) Burst Astrophysics. Journal of High Energy Physics, Gravitation and Cosmology, 3, 157-166. https://doi.org/10.4236/jhepgc.2017.32016

[7] Netchitailo, V. (2017) Mathematical Overview of Hypersphere World-Universe Model. Journal of High Energy Physics, Gravitation and Cosmology, 3, 415-437. https://doi.org/10.4236/jhepgc.2017.33033

[8] Netchitailo, V. (2017) Astrophysics: Macroobject Shell Model. Journal of High Energy Physics, Gravitation and Cosmology, 3, 776-790. https://doi.org/10.4236/jhepgc.2017.34057

[9] Netchitailo, V. (2018) Analysis of Maxwell's Equations. Cosmic Magnetism. Journal of High Energy Physics, Gravitation and Cosmology, 4, 1-7. https://doi.org/10.4236/jhepgc.2018.41001

[10] Netchitailo, V. (2018) Hypersphere World-Universe Model. Tribute to Classical Physics. Journal of High Energy Physics, Gravitation and Cosmology, 4, 441-470. https://doi.org/10.4236/jhepgc.2018.43024

[11] Netchitailo, V. (2019) Solar System. Angular Momentum. New Physics. Journal of High Energy Physics, Gravitation and Cosmology, 5, 112-139. https://doi.org/10.4236/jhepgc.2019.51005

[12] Netchitailo, V. (2019) High-Energy Atmospheric Physics: Ball Lightning. Journal of High Energy Physics, Gravitation and Cosmology, 5, 360-374. https://doi.org/10.4236/jhepgc.2019.52020

[13] Riemann, B. (1854) On the Hypotheses which lie at the Bases of Geometry. Nature, 8, 14-17, 36, 37.

[14] Heaviside, O. (1893) A Gravitational and Electromagnetic Analogy. The Electrician, 31,81 .

[15] Tesla, N. (1937) Prepared Statement on the 81st Birthday Observance. http://www.institutotesla.org/tech/TeslaGravity.html

[16] Dirac, P.M. (1951) Is There an Aether? Nature, 168, 906. 
https://doi.org/10.1038/168906a0

https://web.archive.org/web/20081217042934

[17] Dirac, P.A.M. (1937) The Cosmological Constants. Nature, 139, 323.

https://doi.org/10.1038/139323a0

[18] Hoyle, F. and Narlikar, J.V. (1964) A New Theory of Gravitation. Proceedings of the Royal Society of London, A282, 178.

[19] Dirac, P.A.M. (1974) Cosmological Models and the Large Numbers Hypothesis. Proceedings of the Royal Society of London, A338, 439.

https://doi.org/10.1098/rspa.1974.0095

[20] McCullagh, J. (1846) An Essay towards a Dynamical Theory of Crystalline Reflexion and Refraction. Transactions of the Royal Irish Academy, 21, 17.

[21] Burbidge, E.M., Burbidge, G.R., Fowler, W.A. and Hoyle, F. (1957) Synthesis of the Elements in Stars. Reviews of Modern Physics, 29, 547-650.

https://doi.org/10.1103/RevModPhys.29.547

[22] Kohlrausch, R. and Weber, W. (1857) On the Amount of Electricity Which Flows through the Cross-Section of the Circuit in Galvanic Currents.

http://ppp.unipv.it/Collana/Pages/Libri/Saggi/Volta\%20and\%20the\%20History\%20 of $\% 20$ Electricity/V\%26H\%20Sect3/V\%26H\%20287-297.pdf

[23] Heüman, G.D. (1888) The Rydberg Formula as Presented to Matematiskt-Fysiska Förening. https://commons.wikimedia.org/wiki/File:Rydbergformula.jpg

[24] Thomson, J.J. (1897) Cathode Rays. Philosophical Magazine, 44, 293.

http://web.lemoyne.edu/ giunta/thomson1897.html

https://doi.org/10.1080/14786449708621070

[25] Plank, M. (1901) On the Law of Distribution of Energy in the Normal Spectrum. Annalen der Physik, 4, 553-562. https://doi.org/10.1002/andp.19013090310

[26] Bennett, C.L., et al. (2013) Nine-Year Wilkinson Microwave Anisotropy Probe (WMAP) Observations: Final Maps and Results. The Astrophysical Journal Supplement Series, 208, 20. https://doi.org/10.1088/0067-0049/208/2/20

[27] Zuckerman, B. and Malkan, M.A. (1996) The Origin and Evolution of the Universe. Jones and Bartlet Publishers, Burlington.

https://books.google.com/books?id=G0iR4jpWKN4C\&pg=PA4\&lpg=PA4\&dq=\%22criti cal+density+universe $\% 22+\% 22$ escape+velocity $\% 22 \&$ source=bl\&ots=ym46gfQUpI\& sig=ACfU3U0-2_bRxgpJURIP0Kj44xTq7JHK7w\&hl=en\&sa=X\&ved=2ahUKEwiOaK4lZXhAhUDHDQIHW7_BmYQ6AEwBHoECAkQAQ\#v=onepage\&q=\%22critic al\%20density $\% 20$ universe $\% 22 \% 20 \% 22$ escape $\% 20$ velocity $\% 22 \& \mathrm{f}=$ false

[28] Mirizzi, A., Raffelt, G.G. and Serpico, P.D. (2006) Photon-Axion Conversion in Intergalactic Magnetic Fields and Cosmological Consequences. arXiv: 0607415.

[29] Keane, E.F., et al. (2016) A Fast Radio Burst Host Galaxy. Nature, 530, 453-456.

[30] Fixsen, D.J. (2009) The Temperature of the Cosmic Microwave Background. The Astrophysical Journal, 707, 916. https://doi.org/10.1088/0004-637X/707/2/916

[31] Bonetti, L., et al. (2017) FRB 121102 Casts New Light on the Photon Mass. Physics Letters B, 768, 326-329. https://doi.org/10.1016/j.physletb.2017.03.014

[32] Sanchez, M. (2003) Oscillation Analysis of Atmospheric Neutrinos in Soudan 2. PhD Thesis, Tufts University, Medford. http://nu.physics.iastate.edu/Site/Bio_files/thesis.pdf

[33] Kaus, P. and Meshkov, S. (2003) Neutrino Mass Matrix and Hierarchy. AIP Conference Proceedings, 672, 117. https://doi.org/10.1063/1.1594399

[34] Battye, R.A. and Moss, A. (2014) Evidence for Massive Neutrinos from CMB and 
Lensing Observations. Physical Review Letters, 112, Article ID: 051303. https://doi.org/10.1103/PhysRevLett.112.051303

[35] Maurette, M., Cragin, J. and Taylor, S. (1992) Cosmic Dust in 50 KG Blocks of Blue Ice from Cap-Prudhomme and Queen Alexandra Range, Antarctica. Meteoritics, 27, 257. https://doi.org/10.1111/j.1945-5100.1992.tb00233.x

[36] Saxton, J.M., Knotts, S.F., Turner, G. and Maurette, M. (1992) 40Ar/39Ar Studies of Antarctic Micrometeorites. Meteoritics, 27, 285.

[37] Jackson, A.A. and Zook, H.A. (1991) Dust Particles from Comets and Asteroids: Parent-Daughter Relationships. Abstracts of the Lunar and Planetary Science Conference, 22, 629-630.

[38] Lagache, G., et al. (1999) First Detection of the Warm Ionized Medium Dust Emission. Implication for the Cosmic Far-Infrared Background. arXiv: 9901059.

[39] Finkbeiner, D.P., Davis, M. and Schlegel, D.J. (2000) Detection of a Far IR Excess with DIRBE at 60 and 100 Microns. International Astronomical Union, the Extragalactic Infrared Background and Its Cosmological Implication, Volume 204, 121. https://doi.org/10.1017/S0074180900225953

[40] Siegel, P.H. (2002) Terahertz Technology. IEEE Transactions on Microwave Theory and Techniques, 50, 910-928. https://doi.org/10.1109/22.989974

[41] Phillips, T.G. and Keene, J. (1992) Submillimeter Astronomy [Heterodyne Spectroscopy]. Proceedings of the IEEE, 80, 1662. https://doi.org/10.1109/5.175248

[42] Dupac, X., et al. (2003) The Complete Submillimeter Spectrum of NGC 891. Monthly Notices of the Royal Astronomical Society, 344, 105-109. https://doi.org/10.1046/j.1365-8711.2003.06793.x

[43] Aguirre, J.E., et al. (2003) The Spectrum of Integrated Millimeter Flux of the Magellanic Clouds and 30-Doradus from TopHat and DIRBE Data. The Astrophysical Journal, 596, 273. https://doi.org/10.1086/377601

[44] Pope, A., et al. (2006) Using Spitzer to Probe the Nature of Submillimetre Galaxies in GOODS-N. arXiv: 0603409.

[45] Marshall, J.A., et al. (2007) Decomposing Dusty Galaxies. I. Multi-Component Spectral Energy Distribution Fitting. arXiv: 0707.2962.

[46] Devlin, M.J., et al. (2009) Over Half of the Far-Infrared Background Light Comes from Galaxies at $\mathrm{z}>=1.2$. Nature, 458, 737-739.

https://doi.org/10.1038/nature07918

[47] Chapin, E.L., et al. (2010) A Joint Analysis of BLAST 250-500um and LABOCA 870um Observations in the Extended Chandra Deep Field South. Monthly Notices of the Royal Astronomical Society, 411, 505-549. https://doi.org/10.1111/j.1365-2966.2010.17697.x

[48] Mackenzie, T., et al. (2010) A Pilot Study for the SCUBA-2 “All-Sky" Survey. arXiv: 1012.1655 .

[49] Serra, P., et al. (2014) Cross-Correlation of Cosmic Infrared Background Anisotropies with Large Scale Structures. Astronomy and Astrophysics, 570, A98. https://doi.org/10.1051/0004-6361/201423958

[50] Lee, B.W. and Weinberg, S. (1977) Cosmological Lower Bound on Heavy-Neutrino Masses. Physical Review Letters, 39, 165-168. https://doi.org/10.1103/PhysRevLett.39.165

[51] Dicus, D.A., Kolb, E.W. and Teplitz, V.L. (1977) Cosmological Upper Bound on Heavy-Neutrino Lifetimes. Physical Review Letters, 39, 168-171.

https://doi.org/10.1103/PhysRevLett.39.168 
[52] Dicus, D.A., Kolb, E.W. and Teplitz, V.L. (1978) Cosmological Implications of Massive, Unstable Neutrinos. The Astrophysical Journal, 221, 327-341. https://doi.org/10.1086/156031

[53] Gunn, J.E., et al. (1978) Some Astrophysical Consequences of the Existence of a Heavy Stable Neutral Lepton. The Astrophysical Journal, 223, 1015-1031. https://doi.org/10.1086/156335

[54] Stecker, F.W. (1978) The Cosmic Gamma-Ray Background from the Annihilation of Primordial Stable Neutral Heavy Leptons. The Astrophysical Journal, 223, 1032-1036. https://doi.org/10.1086/156336

[55] Zeldovich, Ya.B., Klypin, A.A., Khlopov, M.Yu. and Chechetkin, V.M. (1980) Astrophysical Constraints on the Mass of Heavy Stable Neutral Leptons. Soviet Journal of Nuclear Physics, 31, 664-669.

[56] Spolyar, D., Freese, K. and Gondolo, P. (2007) Dark Matter and the First Stars: A New Phase of Stellar Evolution. AIP Conference Proceedings, 990, 42. https://doi.org/10.1063/1.2905656

[57] Freese, K., Rindler-Daller, T., Spolyar, D. and Valluri, M. (2015) Dark Stars: A Review. Reports on Progress in Physics, 79, Article ID: 066902. https://doi.org/10.1088/0034-4885/79/6/066902

[58] Corda, C. (2009) Interferometric Detection of Gravitational Waves: The Definitive Test for General Relativity. International Journal of Modern Physics, 18, 2275-2282. https://doi.org/10.1142/S0218271809015904

[59] Boehm, C., Fayet, P. and Silk, J. (2003) Light and Heavy Dark Matter Particles. Physical Review D, 69, 101302(R). https://doi.org/10.1103/PhysRevD.69.101302

[60] Mehrgan, K., et al. (2019) A 40-Billion Solar Mass Black Hole in the Extreme Core of Holm 15A, the Central Galaxy of Abell 85. arXiv:1907.10608.

[61] NASA (2015) The Cosmic Distance Scale. https://imagine.gsfc.nasa.gov/features/cosmic/local_supercluster_info.html

[62] Tully, R.B. (1982) The Local Supercluster. Astrophysical Journal, 257, 389. https://doi.org/10.1086/159999

[63] Heymans, C., et al. (2008) The Dark Matter Environment of the Abell 901/902 Supercluster: A Weak Lensing Analysis of the HST STAGES Survey. Monthly Notices of the Royal Astronomical Society, 385, 1431-1442. https://doi.org/10.1111/j.1365-2966.2008.12919.x

[64] Zwicky, F. (1933) Die Rotverschiebung von extragalaktischen Nebeln. Helvetica Physica Acta, 6, 110.

[65] Ness, M., et al. (2015) The Cannon: A Data-Driven Approach to Stellar Label Determination. The Astrophysical Journal, 808, 16. https://doi.org/10.1088/0004-637X/808/1/16

[66] Bond, H.E., et al. (2013) HD 140283: A Star in the Solar Neighborhood that Formed Shortly After the Big Bang. arXiv:1302.3180.

[67] Marchetti, T., Rossi, E.M. and Brown, A.G.A. (2018) Gaia DR2 in 6D: Searching for the Fastest Stars in the Galaxy. Monthly Notices of the Royal Astronomical Society, sty2592. https://doi.org/10.1093/mnras/sty2592

[68] Koposov, S.E., et al. (2019) The Great Escape: Discovery of a Nearby $1700 \mathrm{~km} / \mathrm{s} \mathrm{Star}$ Ejected from the Milky Way by Sgr A*. arXiv:1907.11725.

[69] Clarke, C.J., et al. (2018) High-Resolution Millimeter Imaging of the CI Tau Protoplanetary Disk: A Massive Ensemble of Protoplanets from 0.1 to 100 au. The $A s$ trophysical Journal Letters, 866, L6. https://doi.org/10.3847/2041-8213/aae36b 
[70] Wright, E.L. (2001) Cosmic InfraRed Background Radiation. http://www.astro.ucla.edu/ wright/CIBR

[71] Ansoldi, S., et al. (2015) Teraelectronvolt Pulsed Emission from the Crab Pulsar Detected by MAGIC. arXiv: 1510.07048 .

[72] Chen, G., et al. (2015) NuSTAR Observations of the Young, Energetic Radio Pulsar PSR B1509-58. The Astrophysical Journal, 817, 93. https://doi.org/10.3847/0004-637X/817/2/93

[73] Johnston, S., et al. (1993) Discovery of a Very Bright, Nearby Binary Millisecond Pulsar. Nature, 361, 613-615. https://doi.org/10.1038/361613a0

[74] Bailes, M., et al. (1994) Discovery of Three Binary Millisecond Pulsars. The Astrophysical Journal, 425, L41-L44. https://doi.org/10.1086/187306

[75] Pletsch, H.J., et al. (2012) Binary Millisecond Pulsar Discovery via Gamma-Ray Pulsations. Science, 338, 1314-1317. https://doi.org/10.1126/science.1229054

[76] Boyajian, T.S., et al. (2015) Planet Hunters X. KIC 8462852-Where's the Flux? arXiv:1509.03622.

[77] Scaringi, S., et al. (2016) The Peculiar Dipping Events in the Disk-Bearing Young-Stellar Object EPIC 204278916. Monthly Notices of the Royal Astronomical Society, 463, 2265-2272. https://doi.org/10.1093/mnras/stw2155

[78] Ansdell, M., et al. (2015) Young "Dipper" Stars in Upper Sco and $\rho$ Oph Observed by K2. The Astrophysical Journal, 816, 69. https://doi.org/10.3847/0004-637X/816/2/69

[79] Ansdell, M., et al. (2016) Dipper Disks Not Inclined towards Edge-On Orbits. arXiv: 1607.03115.

[80] Rappaport, S., et al. (2019) Deep Long Asymmetric Occultation in EPIC 204376071. Monthly Notices of the Royal Astronomical Society, 485, 2681. https://doi.org/10.1093/mnras/stz537

[81] Opher, M., et al. (2015) Magnetized Jets Driven by the Sun: The Structure of the Heliosphere Revisited. The Astrophysical Journal Letters, 800, L28. https://doi.org/10.1088/2041-8205/800/2/L28

[82] Aguilar, D.A. and Pulliam, C. (2010) Astronomers Find Giant, Previously Unseen Structure in Our Galaxy. Harvard-Smithsonian Center for Astrophysics. Release No. 2010-22.

[83] Yang, L. and Razzaque, S. (2019) Constraints on Very High Energy Gamma-Ray Emission from the Fermi Bubbles with Future Ground-Based Experiments. Physical Review D, 99, Article ID: 083007. https://doi.org/10.1103/PhysRevD.99.083007

[84] Su, M. and Finkbeiner, D.P. (2012) Evidence for Gamma-Ray Jets in the Milky Way. The Astrophysical Journal, 753, 61. https://doi.org/10.1088/0004-637X/753/1/61

[85] Ponti, G., et al. (2019) An X-Ray Chimney Extending Hundreds of Parsecs above and below the Galactic Centre. Nature, 567, 347-350.

https://doi.org/10.1038/s41586-019-1009-6

[86] Hooper, D. and Slatyer, T.R. (2013) Two Emission Mechanisms in the Fermi Bubbles: A Possible Signal of Annihilating Dark Matter. Physics of the Dark Universe, 2, 118-138. https://doi.org/10.1016/j.dark.2013.06.003

[87] Hooper, D. and Goodenough, L. (2011) Dark Matter Annihilation in the Galactic Center as Seen by the Fermi Gamma Ray Space Telescope. Physics Letters B, 697, 412. https://doi.org/10.1016/j.physletb.2011.02.029

[88] McDaniel, A., Jeltema, T. and Profumo, S. (2018) A Multi-Wavelength Analysis of Annihilating Dark Matter as the Origin of the Gamma-Ray Emission from M31. 
Physical Review D, 97, Article ID: 103021. https://doi.org/10.1103/PhysRevD.97.103021

[89] Yang, H.Y.K., Ruszkowski, M. and Zweibel, E.G. (2018) Unveiling the Origin of the Fermi Bubbles. Galaxies, 6, 29. https://doi.org/10.3390/galaxies6010029

[90] Beall, J.H. (2015) A Review of Astrophysical Jets. Acta Polytechnica CTU Proceedings, 1, 259-264.

[91] Swedenborg, E. (1734) Philosophical and Mineralogical Works. Principia, 1. https://www.milestone-books.de/pages/books/002966/emanuel-swedenborg/operaphilosophica-et-mineralia-volumes-i-iii-all-published

[92] Brush, S.G. (2014) A History of Modern Planetary Physics: Nebulous Earth. 7.

[93] Darwin, G.H. (1879) On the Bodily Tides of Viscous and Semi-Elastic Spheroids, and on the Ocean Tides upon a Yielding Nucleus. Philosophical Transactions of the Royal Society, 170, 1-35. https://doi.org/10.1098/rstl.1879.0061

[94] Wise, D.U. (1966) Origin of the Moon by Fission. In: Marsden, B.G. and Cameron, A.G.W., Eds., Proceedings of an International Conference, Plenum Press, New York, 213. http://adsabs.harvard.edu/full/1966ems..conf..213W https://doi.org/10.1007/978-1-4684-8401-4_14

[95] Jacot, L. (1986) Heretical Cosmology (Transl. of Science et bon sense, 1981). Exposition-Banner.

[96] Van Flandern, T. (1999) Dark Matter, Missing Planets, and New Comets. North Atlantic Books, Berkeley.

[97] Djorgovski, S.G. (2016) Stellar Structure and the Sun. http://www.astro.caltech.edu/ george/ay1/lec_pdf/Ay1_Lec08.pdf

[98] Dziewonski, A.M. anderson, D.L. (1981) Preliminary Reference Earth Model. Physics of the Earth and Planetary Interiors, 25, 297. https://doi.org/10.1016/0031-9201(81)90046-7

[99] Fossat, E., et al. (2017) Asymptotic g Modes: Evidence for a Rapid Rotation of the Solar Core. Astronomy and Astrophysics, 604, A40. https://doi.org/10.1051/0004-6361/201730460

[100] Zhang, J., et al. (2005) Inner Core Differential Motion Confirmed by Earthquake Waveform Doublets. Science, 309, 1357-1360. https://doi.org/10.1126/science.1113193

[101] Cole, G.H.A. and Woolfson, M.M. (2002) Planetary Science: The Science of Planets around Stars. Institute of Physics Publishing, Bristol, 36-37, 380-382. https://doi.org/10.1887/075030815X

[102] Kinver, M. (2009) Global Average Temperature May Hit Record Level in 2010. BBC.

[103] Pollack, H.N., Hurter, S.J. and Johnson, J.R. (1993) Heat Flow from the Earth's Interior: Analysis of the Global Data Set. Reviews of Geophysics, 31, 267-280. https://doi.org/10.1029/93RG01249

[104] Arculus, R. (2016) The Cosmic Origins of Uranium. http://www.world-nuclear.org/information-library/nuclear-fuel-cycle/uranium-reso urces/the-cosmic-origins-of-uranium.aspx

[105] Gando, A., et al. (2011) Partial Radiogenic Heat Model for Earth Revealed by Geoneutrino Measurements. Nature Geoscience, 4, 647-651. https://doi.org/10.1038/ngeo1205

[106] Hoffman, D.C., et al. (1971) Detection of Plutonium-244 in Nature. Nature, 234, 132-134. https://doi.org/10.1038/234132a0 
[107] Ricard, Y. (2009) Physics of Mantle Convection. In: Bercovici, D. and Schubert, G., Eds., Treatise on Geophysics. Mantle Dynamics, Elsevier Science, Amsterdam, 7.

[108] Elkins-Tanton, L.T. (2006) Jupiter and Saturn. Chelsea House, New York.

[109] O’Donoghue, J., Moore, L., Stallard, T.S. and Melin, H. (2016) Heating of Jupiter's Upper Atmosphere above the Great Red Spot. Nature, 536, 190-192. https://doi.org/10.1038/nature18940

[110] De Pater, I. and Lissauer, J.J. (2010) Planetary Sciences. 2nd Edition, Cambridge University Press, Cambridge, 254-255.

[111] (2004) Class 12-Giant Planets-Heat and Formation. 3750-Planets, Moons \& Rings. Colorado University, Boulder.

[112] Pearl, J.C. and Conrath, B.J. (1991) The Albedo, Effective Temperature, and Energy Balance of Neptune, as Determined from Voyager Data. Journal of Geophysical Research: Space Physics, 96, 18921-18930. https://doi.org/10.1029/91JA01087

[113] Kamata, S., et al. (2019) Pluto's Ocean Is Capped and Insulated by Gas Hydrates. Nature Geoscience, 12, 407-410. https://doi.org/10.1038/s41561-019-0369-8

[114] Feulner, G. (2012) The Faint Young Sun Problem. Reviews of Geophysics, 50, RG2006. https://doi.org/10.1029/2011RG000375

[115] Hoyle, F. (1958) Remarks on the Computation of Evolutionary Tracks. Ricerche Astronomiche, 5, 223.

[116] Schwarzschild, M. (1958) Structure and Evolution of the Stars. Princeton University Press, Princeton. https://doi.org/10.1515/9781400879175

[117] Newman, M.J. and Rood, R.T. (1977) Implications of Solar Evolution for the Earth's Early Atmosphere. Science, 198, 1035-1037. https://doi.org/10.1126/science.198.4321.1035

[118] Gough, D.O. (1981) Solar Interior Structure and Luminosity Variations. Solar Physics, 74, 21-34. https://doi.org/10.1007/BF00151270

[119] Baryshev, Yu. (2008) Field Fractal Cosmological Model as an Example of Practical Cosmology Approach. arXiv: 0810.0162.

[120] Agle, D.C. and Brown, D. (2012) Data from NASA's Voyager 1 Point to Interstellar Future. http://www.nasa.gov/mission_pages/voyager/voyager20120614.html

[121] Abhyankar, K.D. (1977) A Survey of the Solar Atmospheric Models. Bulletin of the Astronomical Society of India, 5, 40-44.

[122] Fox, K.C. (2014) NASA's STEREO Maps Much Larger Solar Atmosphere than Previously Observed.

https://www.nasa.gov/content/goddard/nasas-stereo-maps-much-larger-solar-atmo sphere-than-previously-observed

[123] Aschwanden, M.J. (2004) Physics of the Solar Corona. An Introduction. Praxis Publishing, Singapore.

[124] Schmelz, J.T., et al. (2012) Composition of the Solar Corona, Solar Wind, and Solar Energetic Particles. The Astrophysical Journal, 755, 33.

https://doi.org/10.1088/0004-637X/755/1/33 http://iopscience.iop.org/article/10.1088/0004-637X/755/1/33/pdf

[125] Grossman, L. (2018) Strange Gamma Rays from the Sun May Help Decipher Its Magnetic Fields. Science News, 194, 9.

https://www.sciencenews.org/article/strange-gamma-rays-sun-magnetic-fields

[126] Baliukin, I.I., et al. (2019) SWAN/SOHO Lyman- $\alpha$ Mapping: The Hydrogen Geocorona Extends Well beyond the Moon. JGR Space Physics, 124, 861-885. 
https://doi.org/10.1029/2018JA026136

[127] Reyes, R. (2012) Exploring the Sun-Earth Connection. Southwest Research Institute, San Antonio. http://pluto.space.swri.edu/image/glossary/geocorona.html

[128] Kameda, S., et al. (2017) Ecliptic North-South Symmetry of Hydrogen Geocorona. Geophysical Research Letter, 44, 11706-11712. https://doi.org/10.1002/2017GL075915

[129] Kuwabara, M., et al. (2017) The Geocoronal Responses to the Geomagnetic Disturbances. Journal of Geophysical Research: Space Physics, 122, 1269-1276. https://doi.org/10.1002/2016JA023247 https://agupubs.onlinelibrary.wiley.com/doi/pdf/10.1002/2016JA023247

[130] NASA (2012) Solar System. http://chandra.harvard.edu/xray_sources/solar_system.html

[131] Wargelin, B.J., et al. (2014) Observation and Modeling of Geocoronal Charge Exchange X-Ray Emission During Solar Wind Gusts. The Astrophysical Journal, 796, Article ID: 28. https://doi.org/10.1088/0004-637X/796/1/28

[132] Cravensa, T.E., et al. (2009) Solar Wind Charge Exchange Contributions to the Diffuse X-Ray Emission. AIP Conference Proceedings, 1156, 37-51. https://doi.org/10.1063/1.3211832

[133] Shematovich, V.I. and Bisikalo, D.V. (2018) Hot Planetary Coronas. Planetary Science. https://doi.org/10.1093/acrefore/9780190647926.013.104 http://planetaryscience.oxfordre.com/view/10.1093/acrefore/9780190647926.001.00 01/acrefore-9780190647926-e-104

[134] Dwyer, J.R. (2012) The Mystery of Lightning. http://www.insightcruises.com/events/sa24/PDF/The_Mysteries_of_Lightning.pdf

[135] Gurevich, A.V., Milikh, G.M. and Roussel-Dupre, R. (1992) Runaway Electron Mechanism of Air Breakdown and Preconditioning during a Thunderstorm. Physics Letters A, 165, 463-468. https://doi.org/10.1016/0375-9601(92)90348-P

[136] Fishman, G.J., et al. (1994) Discovery of Intense Gamma-Ray Flashes of Atmospheric Origin. Science, 264, 1313-1316. https://doi.org/10.1126/science.264.5163.1313

[137] (2019) Russian Satellite Registers Unknown Physical Phenomena in Earth's Atmosphere.

https://sputniknews.com/science/201902111072298631-russia-satellite-unknown-ph ysical-phenomena-earth-atmosphere

[138] Tesla, N. (1904) The Transmission of Electrical Energy without Wires. Electrical World and Engineer.

http://web.archive.org/web/20051222121927/http://tfcbooks.com/tesla/wireless01.ht $\underline{\mathrm{m}}$

[139] (1988) Tesla and Ball Lightning. https://www.bibliotecapleyades.net/tesla/esp_tesla_20.htm

[140] Kapitsa, P.L. (1955) The Nature of Ball Lightning. In: Ritchie, D.J., Ed., Ball Lightning: A Collection of Soviet Research in English Translation (1961 ed.), Consultants Bureau, New York, 11-16.

[141] Handel, P.H. (1975) Maser Theory of Ball Lightning. Bulletin of the American Physical Society Series II, 20, 26.

[142] Ashby, D.E.T.F. and Whitehead, C. (1971) Is Ball Lightning Caused by Antimatter Meteorites? Nature, 230, 180-182. https://doi.org/10.1038/230180a0

[143] Rabinowitz, M. (2002) Little Black Holes: Dark Matter and Ball Lightning. arXiv: 
0212251.

[144] Thornhill, W. (2006) The IEEE, Plasma Cosmology and Extreme Ball Lightning. https://www.holoscience.com/wp/the-ieee-plasma-cosmology-and-extreme-ball-lig htning

[145] Van Devender, P. (2011) Extreme Ball Lightning: New Physics, New Energy Source, or Just Great Fun. https://www.osti.gov/biblio/1107768

[146] Wu, H.C. (2014) Theory of Ball Lightning. arXiv: 1411.4784.

[147] Oreshko, A.G. (2012) Observation of Dark Spherical Area after Passage of Ball Lightning through Thick Absorbers.

https://www.researchgate.net/profile/Alexander_Oreshko/publication/312218738_Observ ation_of_Dark_Spherical_Area_After_Passage_of_Ball_Lightning_Through_Thick_Abso rbers/links/5877307808ae329d6226e786/Observation-of-Dark-Spherical-Area-After -Passage-of-Ball-Lightning-Through-Thick-Absorbers.pdf

[148] Cen, J., Yuan, P. and Xue, S. (2014) Observation of the Optical and Spectral Characteristics of Ball Lightning. Physical Review Letters, 112, Article ID: 035001.

https://doi.org/10.1103/PhysRevLett.112.035001 